\title{
1 Spatiotemporal dynamics of the calcineurin target CrzA
}

2

3

4 Patricia Hernández-Ortiz ${ }^{1}$ and Eduardo A. Espeso*

5 Department of Cellular and Molecular Biology, Centro de Investigaciones Biológicas, CSIC,

6 Madrid, Spain

7

8 Running Head: CrzA dynamics in Aspergillus nidulans

9

10 * Corresponding author, eespeso@cib.csic.es

$11{ }^{1}$ Present address: Manchester Fungal Infection Group, Institute of Inflammation and Repair,

12 CTF Building, Grafton Street, University of Manchester, Manchester, United Kingdom. 
15

\section{Highlights}

- Signaling of CrzA occurs in a basipetal, tip to nucleus, manner and dependent on the apical localization of calmodulin and calcineurin together with actin cytoskeleton.

- CrzA is the only element of its signaling pathway that changes in localization after calcium or alkaline $\mathrm{pH}$ stress.

- Calcineurin is the key factor in the spatiotemporal dynamics of CrzA in the fungal cell.

- Timing of nuclear localization of CrzA depends on the strength and nature of ambient stimulus.

3

\section{Abstract}

6 The response of Aspergilli to elevated concentrations of extracellular calcium and manganese, or environmental alkalinization is mediated by CrzA, a calcineurin-responsive transcription factor (TF). CrzA is the effector of a signaling pathway which includes the apical proteins calmodulin and calcineurin, and the protein kinases GskA and CkiA. Preferentially located in the cytoplasm, CrzA is the only element of the pathway modifying its localization under those stress conditions, being imported into nuclei. Remarkably, there is a direct relationship between the nature/intensity of the stimulus and the pace of nuclear import and time of nuclear permanence of CrzA. Alkalinity caused a transient nuclear accumulation of CrzA while high $\mathrm{Ca}^{2+}$ and $\mathrm{Mn}^{2+}$ concentrations generated a long-lasting accumulation. Furthermore, $\mathrm{Ca}^{2+}$ concentrations (below $5 \mathrm{mM}$ ) that are non-toxic for a $c r z A \Delta$ mutant promoted full signaling of CrzA. However, micromolar concentrations or a mutation disrupting the interaction of CrzA with the phosphatase complex calcineurin, permitted the visualization of a transient and polarized nuclear accumulation of the TF in a tip-to-base gradient. Overall, these results support a model in which nucleo-cytoplasmic dynamics and transcriptional activity of CrzA are driven 
40 by apical signals transmitted by calmodulin and calcineurin. This communication is essential to 41 understand $\mathrm{Ca}^{+2}$-induced stress response in fungi.

42

43 Keywords: Filamentous fungi, calcineurin, calcium signaling, nucleo-cytoplasmic trafficking, 44 transcription factor

45 
Temporal gradient of nuclear $\mathrm{CrzA}$ in response to stress

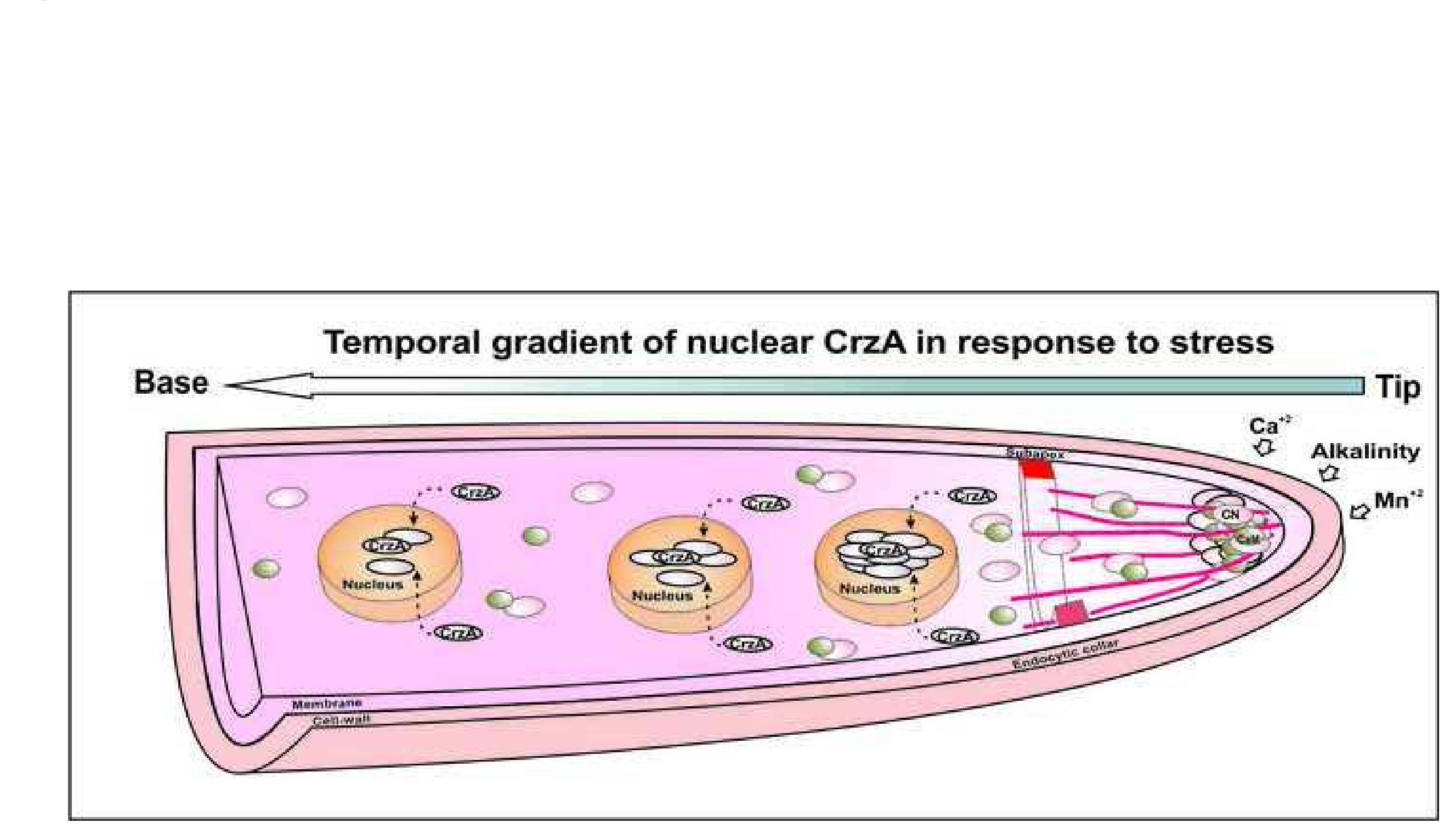

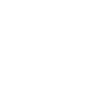

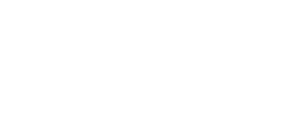

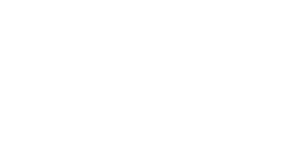

(2)

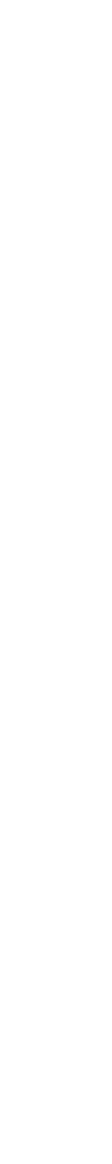

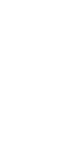

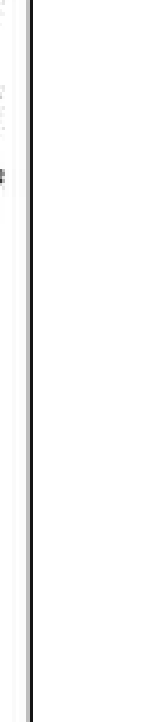




\section{Introduction}

47 Calcium, $\mathrm{Ca}^{2+}$, is an essential secondary component in transduction of many environmental signals but also it is an important source of stress because inappropriate management of intracellular stores may lead to cell death [1]. Intracellular $\mathrm{Ca}^{2+}$ concentrations change in response to environmental and physiological signals, which are followed by the adaptation of the organisms to the new conditions. A ubiquitous and essential $\mathrm{Ca}^{2+}$-signaling mediator protein in eukaryotic cells is calmodulin (CaM) [2]. Under conditions causing high cytosolic $\mathrm{Ca}^{2+}$ concentrations, $\mathrm{CaM}$ binds four $\mathrm{Ca}^{2+}$ atoms inducing structural rearrangements and promoting the interaction of $\mathrm{CaM}$ with its effectors $[3,4]$. One of those effectors is the serine/threonine protein phosphatase calcineurin $(\mathrm{CN})$ [5]. $\mathrm{CN}$ is ubiquitously expressed in eukaryotes and is composed of a catalytic A subunit (CNA) and a regulatory B subunit (CNB) [6]. CN activity requires the recognition of its substrates through a Calcineurin Docking Domain (CDD) composed mainly of non-polar residues following the consensus sequences PxIxIT or LxVP [7, 8].

Although both CaM and $\mathrm{CN}$ are highly conserved proteins in lower and higher eukaryotes, the downstream effectors of this signaling pathway are not as well conserved. Among CN substrates is the Nuclear Factor of Activated T cells (NFAT) in higher eukaryotes and the Calcineurin-Responsive-Zinc-fingers (CRZ) in the fungal kingdom $[9,10,11]$. NFATs are a conserved family of transcription factors (TF) that play important roles in the immune response and in the cardiovascular and nervous systems $[12,13]$, whereas CRZ proteins comprise a family of TFs playing key roles in fungal tolerance to abiotic stresses and in pathogenicity [11, 14]. The phosphorylation state of these TFs regulates their cellular localization and activity. Phosphorylation is associated with cytoplasmic localization $[11,12]$ while their $\mathrm{CN}$-induced dephosphorylation under increased $\mathrm{Ca}^{+2}$ levels promotes nuclear import $[11$, 12].Phosphorylation of fungal CRZs is carried out by different kinases. CK1, Hrr25p and PKA are responsible for phosphorylation of Crzlp in Saccharomyces cerevisiae while a CK1 
homologue CkiA and the GSK3 $\beta$ homologue GskA participate in phosphorylation of CrzA in the filamentous fungus Aspergillus nidulans $[15,16,17]$.

The activity of the CaM-CN-CrzA pathway in filamentous fungi has been adapted to fit in with their growth-mechanism. The characteristic cell-type is the vegetative hypha, which continuously elongate through directed deposition of cell wall and plasmatic membrane materials at one of the apices of the cell [18]. CaM and $\mathrm{CN}$ are apical $[19,20,21]$, while the proteins kinase CkiA is nuclear [22] and GskA is cytoplasmic (this work). All are key elements in the control of cell-growth and this process is associated with the presence of an elevated $\mathrm{Ca}^{2+}$ concentration at the hyphal tip [23]. Thus, the study of this pathway is important to understand how filamentous fungi can grow and thrive under changing environments. Furthermore, the largeness of the apical compartment of a hypha (the region between the growing-apex and the closest nucleus) is a morphological characteristic that facilitates studies of long-distance signaling in eukaryotes. Both features made us use A. nidulans as a model organism to understand long-distance propagation of the calcium signaling pathway. Our results indicate that CrzA is the only effector of the pathway capable of modifying its subcellular localization in response to abiotic signals such as high concentrations of $\mathrm{Ca}^{2+}$ or $\mathrm{Mn}^{2+}$ and alkalinity [15]. Nuclear import of CrzA occurs in a tip-to-base gradient, depends on the nature/intensity of the stimulus and is modulated by the additional members of the pathway, being dephosphorylation a major regulatory step. Results suggest that the nuclear import and transcriptional activity of CrzA is induced by signals originating at the tip and transmitted by calmodulin and calcineurin.

\section{Material and methods}

\subsection{Strains and media conditions}

Media, Aspergillus minimal medium (AMM) and complete medium (ACM), and general techniques for transformation, genetic and molecular analyses of $A$. nidulans strains were used as previously described in [15] and references therein. The A. nidulans strains used are listed in 
Table 1. Transformation DNA cassettes were generated following standardized fusion PCR procedures [24] and using the oligonucleotides listed in Table 2. Aspergillus fumigatus pyr $G^{A f}$ or pyro $A^{A f}$ genes were used as prototrophic selection markers. MAD2446 was the recipient

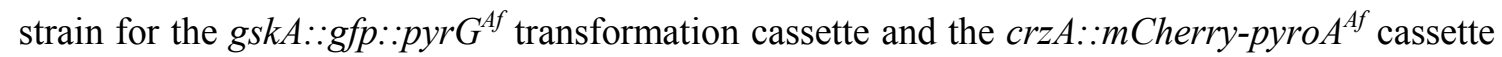
was transformed into recipient strain MAD4366. Integration events were in all cases verified by Southern blotting.

Phenotypic analyses were made by inoculating conidiospores onto solid MMA supplemented with different concentrations of $\mathrm{CaCl}_{2}$ as indicated in the text or with $0.1 \mathrm{M} \mathrm{Na}_{2} \mathrm{HPO}_{4}$ to generate alkaline $\mathrm{pH}(\mathrm{pH} 8)$, and incubated at $37^{\circ} \mathrm{C}$ for 2 days.

\subsection{Protein extraction and Western blot analyses}

The expression of tagged proteins was analyzed by Western blotting following the VFAL method [15]. For total protein extractions, strains were cultivated in supplemented fermentation media for $18 \mathrm{~h}$ at $37^{\circ} \mathrm{C}$, and then $\mathrm{CaCl}_{2}(1 \mathrm{mM}$ or $100 \mathrm{mM})$ was added to the culture and further incubated for time periods ranging from 1 to $30 \mathrm{~min}$, as indicated in the text. Then, mycelia was vacuum filtered using 0.45 - $\mu \mathrm{m}$-pore-size nitrocellulose membranes (Scharlau), frozen in dry ice and lyophilized for $16 \mathrm{~h}$. Protein extraction was carried out by alkaline-lysis (AL) extraction procedure as described in [15]. Samples were resolved in 4-15\% Mini-PROTEAN ${ }^{\circledR}$ TGX $^{\mathrm{TM}}$ precast polyacrylamide gels (Bio-Rad) before transfer to nitrocellulose filters using TransBlot ${ }^{\circledR}$ Turbo $^{\mathrm{TM}}$ Transfer System (Bio-Rad). GFP-tagged proteins were detected using a polyclonal mouse anti-GFP (1/5000; Roche). Peroxidase-conjugated goat anti-mouse IgG immunoglobulin (Jackson Immuno Research Laboratories) was used as a secondary antibody at a 1/4000 dilution. Peroxidase activity was assayed with the Amersham Biosciences ECL kit and luminescence detected and recorded using a LAS3000 imager system (Fuji). Images were analyzed using the Multi Gauge V3.0 software (Fuji) 
A. nidulans cells were cultured in appropriately supplemented watch minimal medium (WMM) [25] and incubated at $25^{\circ} \mathrm{C}$ for 18 hours. After this period, the medium was replaced with fresh WMM adjusted to alkaline $\mathrm{pH}$ with $\mathrm{Na}_{2} \mathrm{HPO}_{4}$ (using $0.1 \mathrm{M}$ and $0.01 \mathrm{M}$ concentrations) or Tris$\mathrm{HCl} \mathrm{pH} 8$ buffer at the concentration of $10 \mathrm{mM}$. Media were also supplemented with cations, calcium as $\mathrm{CaCl}_{2}$ salt at final concentrations of $0.01 \mathrm{mM}, 1 \mathrm{mM}$ and $50 \mathrm{mM}$, and manganese, as $\mathrm{MnCl}_{2}$ salt, at $10 \mathrm{mM}$ or $1 \mathrm{mM}$ concentration. When indicated, cytoskeleton depolymerizing agents Latrunculin B $(100 \mu \mathrm{M})$ and Benomyl $(4.8 \mu \mathrm{g} / \mathrm{ml})$ were added to the replacing medium. In all cases, image acquisition was started immediately after the shift. For all shift experiments a single cell was imaged and after the period of incubation with the stress other cells were visualized to confirm the homogeneity of localization for the fusion protein. These shift experiments were repeated for at least 10 times to ensure a correct description for the localization of each fluorescent protein.

For all microscopy experiments, conidia were germinated on uncoated $\mu$-Slide 8 well (Ibidi $\mathrm{GmbH}$, Germany) for a direct observation of cultures using a Leica DMI-6000b inverted microscope equipped with Normarski and epifluorescence optics. Images were acquired with a Hamamatsu ORCA ER digital camera driven by Metamorph software (Universal Imaging Corporation), using Semrock Brightline GFP-3035B and TXRED-4040B (mCherry) filter sets. Images were processed to a minimum using Metamorph Universal Imaging Corporation) or ImageJ (http://rsb.info.nih.gov/ij/index.html) software.

\section{Results}

\subsection{Cellular distribution of components of the CrzA signaling machinery.}

In order to deepen in the mechanism of signal transmission to CrzA in a filamentous fungus, we first focused on the analysis of the cellular distribution of its signaling components. The catalytic subunit of the protein phosphatase calcineurin, CnaA, and protein kinases CkiA and 
151 GskA were tagged a their respective C-terminus with the green fluorescent protein, GFP.

152 Calmodulin (CaM) localization was also included in this study given its role in calcineurin 153 activation $[2,6]$. With the sole exception of the strain expressing CaM-GFP, which displayed 154 compact morphology and reduced radial extension, the resulting tagged strains showed normal 155 colony growth and conidiation on AMM, indicating that these fluorescent chimaeras were functional (Fig. S1).

157 As expected, in the absence of stress, CrzA-GFP fusion located dispersed along the cytoplasm 158 and excluded from nuclei (Fig. 1, [15]). The CaM-GFP fusion preferentially located at the tip of the cell (asterisk at Fig. 1), at septa (white arrowheads, Fig. 1) and in dot-like accumulations along the cytoplasm (black arrowheads, Fig. 1, [21]). The GFP-tagged CnaA fusion showed a similar localization to CaM (Fig. 1). However, CnaA-GFP accumulated at both sides of the septum, being this distribution in A. nidulans similar to that described for calcineurin in the pathogen A. fumigatus [26]. In contrast to the calcineurin/CaM system, tagged kinases CkiAGFP and GskA-GFP did not accumulate at the cell apex (Fig. 1). As previously noted by Apostolaky et al [22], CkiA-GFP was observed in nuclei and along the cytoplasm but we also observed accumulation of this kinase in septa (Fig. 1). Finally, GskA-GFP was evenly distributed through the cytoplasm and excluded from nuclei and septa (Fig. 1). Results evidenced the distinctive cellular localization patterns of signaling elements of CrzA protein in resting cells, showing a highly polarized localization of calmodulin and calcineurin in contrast to a widespread distribution for CkiA and GskA kinases.

3.2. CrzA is the only member of the pathway modifying its subcellular localization under calcium and alkaline $\mathrm{pH}$ stresses.

174 A further objective was the analysis of the cellular distribution of CaM, CnaA, CkiA and GskA under high calcium concentration or alkaline pH stress. None of the tagged strains presented sensitivity to these stimuli, thus an appropriate functional response was expected (Fig. S1). 
177 Figure 2A shows a co-culture of cells expressing CaM-GFP (MAD4310) along with cells 178 expressing CrzA-GFP (MAD3021) which served as control for the response of CrzA to these 179 stresses. Calmodulin distribution did not change either in response to $50 \mathrm{mM}$ calcium or to 180 alkaline $\mathrm{pH}$ stress remaining accumulated at the tip of the cell, meanwhile, as expected, CrzA responded to both stimuli accumulating in nuclei. Next we focused in the dynamics of calcineurin and a strain co-expressing CnaA-GFP and a monomeric red fluorescence Cherry (mCh) tagged CrzA version (MAD4522) was constructed, allowing a simultaneous localization of both fluorescent proteins under stressing conditions. After calcium and alkaline-stress induction the CrzA-mCh fusion accumulated in nuclei as visualized before for the GFP-tagged CrzA. In contrast to CrzA nuclear import, addition of calcium did not modify CnaA cellular distribution, but reduction in the apical accumulation was observed after alkalinization (Fig. 2B). Notably, nuclear accumulation of CnaA was not observed, indicating that the predicted calcineurin-CrzA complex required for proper signaling of this TF may be generated in the cytoplasm but not imported into nuclei. Finally we analyzed the effects of $\mathrm{Ca}^{2+}$ and alkalinity in the distribution of the protein kinases CkiA and GskA. Both protein kinases maintained their original location under calcium or alkaline $\mathrm{pH}$ stress conditions that is mainly nuclear for CkiA and cytoplasmic for GskA (Fig. 2C and D). Thus, CrzA the only component of the cascade analyzed here which undergoes a major change of cellular localization under calcium or alkaline $\mathrm{pH}$ stress.

3.3. Toxic and non-toxic levels of calcium for a null crzA mutant cause similar effects in the post-translational modification pattern and the cellular distribution of $\mathrm{CrzA}$

199 CrzA function is expected to be dependent on its nuclear accumulation. To correlate 200 functionality and nuclear import of this TF, we evaluated a range of calcium concentrations for 201 toxicity of the null $c r z A$ strain and its effect on the localization and post-translational modifications on CrzA. 
203 A comparative phenotypic analysis was carried out between CrzA-GFP and null crzA strains in 204 order to determine the differences in tolerance to a range of $\mathrm{Ca}^{2+}$ concentrations. Figure $3 \mathrm{~A}$ 205 shows that the null $c r z A$ strain was sensitive to a calcium concentration above $50 \mathrm{mM}$ while below $5 \mathrm{mM}$ grew normally. We then investigated how different concentrations of calcium modulate the pattern of post-translational modifications of CrzA. The electrophoretic mobility of this TF was studied in crude extracts of a strain expressing the CrzA-GFP fusion protein. We previously demonstrated that calcium induced an increase in the electrophoretic mobility of CrzA compared to that observed in resting cells, as a result of CrzA dephosphorylation after calcineurin activation [15]. As shown in Figure 3B, addition of a non-toxic, $1 \mathrm{mM}$, or a highly toxic, $100 \mathrm{mM}$, concentration of $\mathrm{CaCl}_{2}$ promoted an immediate increment in the electrophoretic mobility of CrzA from the first minute after addition of the cation. Gradually, low mobility CrzA forms, interpreted as phosphorylated forms (P-CrzA, Fig. 3B, see also [15]), were newly observed along the analyzed times. Thus, results suggest that low and high concentrations of extracellular calcium promoted similar post-translational modifications of CrzA.

217 Since phosphorylation/dephosphorylation of CrzA is directly associated with its cytoplasmic/nuclear localization, the re-appearance of a phosphorylated form after the described increase in the electrophoretic mobility suggests that the nuclear accumulation of the TF may be transient. To confirm this, dependence of CrzA cellular localization on the ambient calcium concentration was examined. As expected, the toxic $\mathrm{Ca}^{2+}$ concentration of $50 \mathrm{mM}$ to a null $\mathrm{crzA}$ mutant induced the nuclear accumulation of CrzA at 1 minute and remained in nuclei after 30 min (Fig. 3C). As a control, and in agreement with previous results, addition of $10 \mathrm{mM} \mathrm{MnCl}_{2}$, also induced a similar effect in the localization of CrzA-GFP (Fig 3C, see also [15]). When cells expressing CrzA-GFP were treated with $1 \mathrm{mM} \mathrm{CaCl}$, nuclear transport of CrzA was observed within the first minute and this localization was maintained for, at least, 30 minutes as it was visualized before for a higher calcium concentration (Fig. 3D). Thus, both toxic (50 mM) and non-toxic $(1 \mathrm{mM})$ levels of extracellular $\mathrm{Ca}^{2+}$ led to an immediate and long-lasting response of CrzA. A ten times reduction in manganese concentration also induced CrzA nuclear localization 
230 but had a temporal effect (Fig. 3E) becoming nuclei empty of fluorescence after 30 min of 231 exposure to this cation. Taken together these results demonstrate that activity of CrzA is not 232 essential for cell survival at low levels of calcium but its molecular and cellular signaling follow 233 a similar pattern of post-translational modifications to that observed when using higher $\mathrm{Ca}^{2+}$ 234 concentrations. Additionally, calcium is a stronger signal for than manganese for maintaining 235 nuclear localization of CrzA.

\subsection{Alkalinity induces a transient nuclear localization of CrzA.}

238 Alkaline $\mathrm{pH}$ constitutes another major stimulus for the nuclear accumulation of CrzA [15]. In 239 view of the long lasting nuclear accumulation of CrzA in cells treated with millimolar 240 concentrations of calcium $(1 \mathrm{mM})$ or manganese $(10 \mathrm{mM})$, we analyzed the timing of nuclear 241 accumulation under $\mathrm{pH}$ stress. As reported before, an immediate import of CrzA in nuclei was 242 visualized after medium alkalinization, which was induced by adding $100 \mathrm{mM} \mathrm{Na} 2 \mathrm{HPO}_{4}$ buffer

243 (Fig. 4A). However this effect on CrzA localization was transient and nuclei were depleted of 244 fluorescence after $10 \mathrm{~min}$ of treatment (Fig. 4A). To verify that this transient effect was not CrzA-GFP fluorescence was first accumulated in the apical nucleus. Next we determined whether using a lower concentration $\mathrm{Na}_{2} \mathrm{HPO}_{4}$ buffer $(10 \mathrm{mM})$ could cause a similar effect. Interestingly, $10 \mathrm{mM} \mathrm{Na}_{2} \mathrm{HPO}_{4}$ generated a brief moderate accumulation of CrzA in nuclei (Fig. 4C). These results indicated a differential effect of ambient stimuli in the activation of CrzA nuclear import machinery, probably relying in differential transmission of ambient $\mathrm{pH}$ signal by calcineurin to CrzA. 


$$
\operatorname{CrzA}
$$

Calcineurin directly interacts with CrzA through the CDD2 and this motif was found to be required for proper signaling of this $\mathrm{TF}$ in response to calcium and alkaline $\mathrm{pH}$ ( [15] and Fig S2). In agreement with this previous observation, the fluorescent mutant version of CrzA lacking CDD2, CrzA ${ }^{\triangle \mathrm{CDD} 2}$-GFP, remained cytoplasmic after medium alkalinization or calcium addition meanwhile a wild type CrzA-GFP fusion was immediately internalized in the nucleus. However, a CrzA ${ }^{\triangle \mathrm{CDD} 2}$ strain (MAD4234) still displayed tolerance to calcium stress which suggested a residual response from this mutant form of CrzA [15]. To test this hypothesis we analyzed the cellular distribution of $\mathrm{CrzA}^{\Delta \mathrm{CDD} 2}$-GFP after longer periods of incubation of cells at stressing conditions. While nuclear exclusion of the TF was maintained for 30 minutes in an alkaline medium as previous observations (results not shown), a remarkable signaling response was observed after prolonged calcium exposure using a $50 \mathrm{mM}$ concentration of $\mathrm{CaCl}_{2}$. Remarkably, nuclear accumulation of $\mathrm{CrzA}^{\triangle \mathrm{CDD} 2}$ was found to be dependent on the distance of each nucleus from the cell apex. This phenomenon is illustrated in the cell shown in Figure 5. The most apical nucleus was the first to accumulate fluorescence after 8 minutes while rest of nuclei remained empty. Ten minutes later, all nuclei accumulated $\mathrm{CrzA}^{\Delta \mathrm{CDD} 2}$. After 15 minutes we observed nuclear transport in two ways: an increment in nuclear fluorescence was detected in basal nuclei meanwhile nuclear export of CrzA started in apical nuclei. Further incubation allowed the visualization of a progressive nuclear exit of CrzA to the cytoplasm, leading to nuclear exclusion from all nuclei after 30 min of treatment. Therefore, in the absence of a CDD2 site CrzA is still signalized in the presence of elevated concentrations of extracellular calcium exhibiting a strongly polarized and transient distribution pattern of nuclear accumulation.

3.6. Spatiotemporal nuclear accumulation of CrzA is visualized under micromolar concentrations of extracellular calcium. 
281 The fact that a strain expressing $\mathrm{CrzA}^{\Delta \mathrm{CDD} 2}$ revealed a non-synchronized CrzA nuclear import at 282 high calcium concentration levels could be indicating an attenuated signaling of the TF. We 283 aimed to obtain this attenuated response in a wild type CrzA-GFP strain by exposing cells to a 284 minimum calcium stress. A reduction to a $10 \mu \mathrm{M}$ concentration of extracellular calcium allowed 285 the observation of a transient and tip-to-base nuclear accumulation of CrzA, similar to that 286 previously detected when using $10 \mathrm{mM}$ Tris- $\mathrm{HCl} \mathrm{pH} 8$ buffer. This effect is exemplified in the 287 cell shown in Figure 6 in which CrzA-GFP fluorescence was followed in three nuclei, numbered 2881,3 and 5 starting from the tip to the basal region of the cell. Initially all nuclei were empty of 289 fluorescence (time 0 min, Fig 6). One minute after calcium addition nuclear accumulation of 290 CrzA was visible in the first nucleus, meanwhile other nuclei were in different stages of CrzA 291 nuclear import. At minute 6 all nuclei accumulated CrzA. From minute 8 onwards, CrzA 292 nuclear export started in the apical nucleus and it was propagated in the same order than nuclear 293 import was previously observed. Fluorescence distribution along the hypha was homogenous 294 after 30 minutes, most probably indicating the cease of the calcium signaling.

295 Consequently, a non-toxic micromolar concentration of calcium constituted an extracellular 296 signal for the activation of CrzA response and induced a cell tip-dependent nuclear 297 accumulation of CrzA. This low-calcium response along with the previous findings on $298 \mathrm{CrzA}^{\triangle \mathrm{CDD} 2}$ signaling suggests that calcium signaling is originated at the tip of the cell.

\subsection{Calcium and alkaline $\mathrm{pH}$ activation of $\mathrm{CrzA}$ is independent of microtubule cytoskeleton.}

301

302

303

304

305

306

In filamentous fungi owing to their elongated cell shape, microtubule-dependent transport ensures long-range transport of cargos [27]. To test whether the transport of CrzA requires the integrity of microtubule cytoskeleton during signaling, we examined the cellular localization of CrzA after tubulin depolymerization. Treatment of $A$. nidulans with the microtubuledisorganizing agent benomyl causes microtubule disassembly in less than ten minutes and the effects on cell morphology are mainly detected as swellings at the tip of cells [28]. A strain 
expressing a CrzA-GFP protein was incubated with benomyl and the localization of CrzA was followed for fifteen minutes. Despite morphological defects observed at the tip, demonstrating the efficacy of the drug treatment, CrzA cellular localization was not altered remaining at the cytoplasm (Fig. 7A). We then investigated the response of CrzA after stress induction in cells previously treated with benomyl. As shown in Figure 7A, CrzA nuclear accumulation was completed after 1 minute of incubation with $50 \mathrm{mM}$ calcium and this localization continued, at least, for 20 minutes. Also microtubule disruption neither affected nuclear import nor nuclear export of CrzA after medium alkalinization (Fig. 7A). These results strongly suggest that nucleo-cytoplasmic trafficking of CrzA is not a microtubule-based transport mechanism.

\subsection{Disorganization of actin cytoskeleton induces nuclear entry of CrzA in a calcineurin- dependent manner}

We further analyzed the role of tubulin and actin cytoskeletons in the cellular localization of CrzA and the signaling elements CnaA and CaM. Figure 7B shows that CaM-GFP maintains its apical localization in the presence of benomyl when cell tips swells and after recovery of polar growth. In contrast, the apical accumulation of CnaA-GFP was partially lost during tip swelling (Fig 7B, left) and some accretions were still visible, which rapidly aggregated at the tip when polarized growth was recovered (Fig. 7B. right). Treatment with actin depolymerizing agents as latrunculin $\mathrm{B}$ or cytochalasin $\mathrm{A}$ resulted in disassembling of F-actin and the arrest of polar growth [28]. A strain expressing a fluorescent tagged version of the actin binding protein AbpA (AbpA-mRFP, MAD1399) was used as a control of efficacy for these drug treatments. Cells treated with latrunculin B detain apical extension and tip started to swell and, following these morphological alterations, the accumulation of AbpA-mRFP in the tips was lost and an even distribution of fluorescence along the cytoplasm was observed [29] and Fig. 7C). Localization of CaM-GFP at a hyphal tip is disrupted by latrunculin B as shown in Figure 7C. Previous reports showed that the disruption of actin cytoskeleton using CytA altered the normal pattern 
333

334

335

336

337

338

339

340

341

342

343

344

345

346

347

348

349

350

351

352

of CaM localization at hyphal tips in A. nidulans [21]. We also observed that actin cytoskeleton participates in maintenance of $\mathrm{CnaA}$ at the apex without affecting the cytoplasmic dot-like accumulations (Fig. 7C). Treatment of cells with actin depolymerizing agents as latrunculin B or cytochalasin D resulted in the induction of transport of CrzA to nuclei (Fig. 7C, S3). Interestingly, cytochalasin had a similar effect to low levels of extracellular calcium and polarized nuclear entry of CrzA was observed (Fig. S3). These results indicate that actin depolymerization promotes signaling of CrzA in the absence of stress stimuli. Furthermore, calcineurin activity is required for the nuclear recruitment of CrzA after actin disruption. Figure 7C shows that in the mutant cnaA $\Delta$ the nuclear import of CrzA was lost after latrunculin $\mathrm{B}$ treatment.

Finally in order to exclude a potential contribution of the microtubule cytoskeleton in nuclear recruitment of CrzA after disruption of actin cytoskeleton, we treated cells with both cytoskeleton depolymerizing drugs, benomyl and latrunculin B. Cells were first treated for 20 minutes with benomyl to disrupt microtubule cytoskeleton and subsequently with latrunculin B. As indicated above, CrzA-GFP did not modify its distribution in presence of benomyl (Fig. 7D). After addition of latrunculin B to the same culture the TF was then recruited to nuclei (Fig. 7D). These observations suggest that actin filaments and not the might be necessary for maintaining CrzA excluded from the nuclei. In fact, microtubule cytoskeleton is most probably not involved in nuclear import nor export of CrzA. 
353
354

\section{Discussion}

In eukaryotes, a general mechanism to regulate the activity of transcription factors is to control the access to their target sequences at the genome ( [30] and references therein). Among the numerous regulatory pathways subjected to this control mechanism are the $\mathrm{Ca}^{2+}-\mathrm{CN}$ activated TFs, as fungal CRZs and mammalian NFATs [12]. Despite the obvious differences between fungi and mammals most regulatory elements and the signaling process of these TFs are extraordinarily conserved. Here we use the filamentous fungus $A$. nidulans and CrzA as models to understand how a CN-dependent TF is dynamically regulated in a polarized cell. We gained evidence for the role of the tip of a multinucleated filamentous fungal cell as a sensing hub for environmental conditions allowing the transmission of signals from tip to nuclei in an ordered mode in response to calcium and alkaline $\mathrm{pH}$ stresses. These findings are summarized in the molecular and cellular models shown in Figure 8.

Several key steps play a part in modulating the function and cellular localization of CrzA (Fig. 7A). A rapid nuclear accumulation of CrzA occurs under specific ambient conditions including medium alkalinization, elevated concentration of manganese and a range of concentrations for $\mathrm{Ca}^{2+}$ (this work and [15]). For all conditions nuclear accumulation of CrzA is observed within the first minute after addition of the stress. However, permanence of CrzA in nuclei is not uniform and depends on the stimulus. Medium alkalinization had a short effect compared to that caused by an elevated concentration of either $\mathrm{Ca}^{2+}$ or $\mathrm{Mn}^{2+}$. Traffic of CrzA to nucleus is a consequence of a direct effect of $\mathrm{CN}$ activity [15]. There are no experimental evidences of direct effects of alkalinity or $\mathrm{Mn}^{2+}$ on promoting the activity of $\mathrm{CN}$ in vivo, however biochemical studies showed for $\mathrm{Mn}^{2+}$ and other metal cations a cofactor role in activation of CN [31]. In Saccharomyces cerevisiae the stress produced by high levels of $\mathrm{Mn}^{2+}$ or alkalinity promotes an increase in intracellular $\mathrm{Ca}^{2+}$ levels which leads to signaling of Crzlp [32]. In Aspergillus, a reduction of $\mathrm{Ca}^{2+}$ concentration had a direct effect on the timing of nuclear accumulation and alkaline $\mathrm{pH}$ signal is dependent on $\mathrm{CN}$ activity (this work and [15]). Therefore, a conversion of alkalinity and high $\mathrm{Mn}^{2+}$ concentration in an intracellular $\mathrm{Ca}^{2+}$ signal is expected in this fungus. 
380 This $\mathrm{Ca}^{2+}$ signal can be originated either from a release of internal stores or by increasing the 381 influx of $\mathrm{Ca}^{2+}$ from the extracellular medium (Fig. 8B). In Candida albicans, alkaline pH 382 enhances the influx of extracellular $\mathrm{Ca}^{2+}$, rising intracellular $\mathrm{Ca}^{2+}$ levels which activate Crzlp 383 signaling [33].

384 Nuclear CrzA certainly constitutes the functional version of this TF and its timely permanence in the nucleus must guarantee a correct transcriptional regulation of genes under its control (Fig. $8 \mathrm{~A}$ and $[34,35])$. Colonial growth of the $\operatorname{crzA} \Delta$ strain is not affected at $\mathrm{Ca}^{2+}$ concentrations below $5 \mathrm{mM} \mathrm{CaCl}_{2}$ indicating that $\mathrm{CrzA}$ activity is not required at those extracellular $\mathrm{Ca}^{2+}$ levels for colony survival. However, both high and low external $\mathrm{Ca}^{2+}$ concentrations induce posttranslational modifications and nuclear import of CrzA suggesting a possible scenario in which CrzA could play different transcriptional roles at high and low calcium stress conditions. To date, the majority of calcium/Crz-dependent transcriptional profiles have been performed with high levels of calcium $(50-200 \mathrm{mM}$, see $[34,36,37,38])$. Consequently, transcriptomic analyses should consider that gene expression under CrzA regulation may differ when the $\mathrm{CN} / \mathrm{Crz}$ system is activated at stressing or non-stressing calcium conditions. This should answer the question whether a transcriptome can be induced by treatment with high and low external calcium concentration but the magnitude of global gene expression be different.

CrzA is the only element belonging to this pathway with major changes in cellular localization in response to stress (Fig. 8B) but the localization of signaling components may play an important role in regulating CrzA activity. A competition between $\mathrm{CN}$ and protein kinases activities becomes determinant to modify CrzA when cytoplasmic $\mathrm{Ca}^{2+}$ drops from a given concentration (Fig 8A). The presence of casein kinase I, CkiA, in the nucleus points to this kinase as responsible for phosphorylation of CrzA in this organelle and of importance for the recognition of CrzA by the nuclear export machinery. CkiA remains nuclear after alkalinization or high $\mathrm{Ca}^{2+}$ concentrations, and there is no evidence of a $\mathrm{Ca}^{2+}$-modulation of CkiA activity in the nucleus which could suggest differential capacity of this kinase to phosphorylate CrzA under stress. In the cytoplasm, the activity of the GskA kinase may produce different 
407 phosphorylation states in CrzA, which have been detected in this and previous studies (Fig. 8A 408 and [15]). As noted for GSK-3 isoforms on activating NFATc nuclear export [39], the activity 409 of GskA on CrzA may oppose to that of $\mathrm{CN}$ at the cytoplasm, contributing to improvement of 410 nuclear exclusion of this TF in the absence of stress or after a stimulation period. As other 411 glycogen synthase kinase homologues, GskA may also be involved in positively regulating the 412 activity of the $\mathrm{CN}$ regulator RCAN ( $\operatorname{RcnA}$ in $A$. nidulans), with a expected direct consequence 413 in reducing $\mathrm{CN}$ activity [38, 40]. In summary, the activities of both kinases may coordinately 414 ensure the exit of CrzA from nuclei and preclude nuclear transport leading to cytoplasmic 415 accumulation for this TF and its subsequent inactivation.

$416 \mathrm{CaM}$ and $\mathrm{CN}$ preferentially locate at the tip of the cell and at septa, and this localization has 417 been associated with their roles in polarized growth or cell wall integrity pathways $[19,20,21]$. 418 Addition of a high extracellular concentration of $\mathrm{Ca}^{2+}$ did not modify the localization of CaM 419 and $\mathrm{CN}$, although these proteins display dynamic movement along the hypha (not shown). Interestingly the formation of the predicted complex between active $\mathrm{CN}$ and $\mathrm{CrzA}$ was not observed. Besides, the absence of nuclear accumulation of $\mathrm{CN}$ and $\mathrm{CaM}$ suggests that after activation of CrzA this heterocomplex must disassemble and consequently CrzA migrate alone to the nucleus.

Integrity of actin but not tubulin cytoskeleton is required for proper regulation of CrzA localization. Disruption of tubulin cytoskeleton had no effect on the nuclear export mechanism of CrzA in resting cells neither affected nuclear import, since $\mathrm{Ca}^{2+}$ induced the rapid entry of 427 CrzA in nuclei of treated cells. However, disruption of actin cytoskeleton had a major impact on CrzA signaling, inducing its nuclear accumulation in resting cells. The fact that F-actin disassembly on CrzA nuclear localization is $\mathrm{CN}$ dependent, suggests that mislocalization of both $\mathrm{CaM}$ and $\mathrm{CN}$ by actin polymerization agents would be triggering the activation of the signaling pathway. Nevertheless the presence of cytoplasmic $\mathrm{Ca}^{2+}$ would be required in these conditions. Supporting this idea is the finding that in mammalian cells disruption of the actin cytoskeleton results in elevation of intracellular $\mathrm{Ca}^{2+}$ levels leading to a prolonged accumulation 
434 of NFAT in the nucleus [41]. Also in neurons, actin filaments can modify $\mathrm{Ca}^{2+}$-influx through

$435 \mathrm{Ca}^{2+}$ channels at the plasma membrane as well as $\mathrm{Ca}^{2+}$-release from internal stores [42]. This 436 interaction between the actin cytoskeleton and intracellular $\mathrm{Ca}^{2+}$ levels could therefore be 437 considered in A. nidulans as the responsible for nuclear recruitment of CrzA following the disruption of the actin cytoskeleton.

439 Finally, the analysis of CrzA signaling at low $\mathrm{Ca}^{2+}$ concentration revealed the spatiotemporal 440 dynamics of CrzA signaling. A final concentration of $10 \mu \mathrm{M}$ calcium induced a CrzA nuclear recruitment following a tip-to-base pattern. Recently, a new mechanism of communication in the asexual development pathway in A. nidulans has been described in which the TF FlbB shows a tip-to-nucleus migration [43]. That work provided evidence for the strict control in the transmission of the information sensed from the tip to the first apical nucleus. The polarized morphology that hyphae show is therefore essential for apical dominance during vegetative growth, required for asexual development and, as our results suggest, important for the adequate stress response. Our work shows that the intensity of the response correlates with intensity of the signal. Only when low levels of calcium are present, the tip-to-base nuclear accumulation of CrzA was observed. The polarized distribution of the $\mathrm{Ca}^{2+}$ signaling elements upstream in the cascade, $\mathrm{CaM}$ and $\mathrm{CN}$, strongly correlates with this polarized response of CrzA (see Fig.7B). completely abolish CrzA binding to CN ( [15] and Fig. S2), which would debilitate the intensity of the response as observed at a low dose of $\mathrm{Ca}^{2+}$ stress. Again this finding would be consistent with the tip-to-base dependence of $\mathrm{CN}$-mediated signaling of CrzA. We propose the 459 contribution of the pool of $\mathrm{CaM}$ and $\mathrm{CN}$ proteins in the apex to the tip-to-base signaling of 460 CrzA (Fig. 8B). 


\section{Conclusions}

463 Due to the polar mode of growth of filamentous fungi during substrate colonization, hyphal tips 464 are the first region of a mycelium in penetrating a new environment. Consequently, besides 465 sustaining growth, hyphal tips must sense the ambiance to lead to a fast adaptive response. 466 Recent publications support a sensorial role for the hyphal tip and demonstrate its importance in 467 coupling ambient signals with the transcriptional control of pathogenicity and reproduction [43, $46844,45]$. Here we describe a novel aspect of dynamics of CrzA nucleo-cytoplasmic transport, 469 revealing a fine tune signaling of the TF in relation to the stimulus encountered and consistent 470 with a model in which tip-to-base gradient of upstream components generates a polarized 471 signaling of this transcription factor. Apical localization of the calmodulin/calcineurin module 472 maintained by the actin cytoskeleton orchestrates the adequate signaling of CrzA to high or low 473 levels of calcium stress and to ambient alkaline $\mathrm{pH}$ resulting in longer or shorter periods of 474 nuclear accumulation. The differential localizations of GskA and CkiA protein kinases in the 475 fungal cell is probably important for maintaining the correct localization of CrzA, most 476 probably through the modulation of the functionality of the nuclear localization signals in CrzA. 477 These observations increase our understanding of how CrzA mediates its function and how 478 calcium and alkaline $\mathrm{pH}$ signaling occurs in a multinucleated cell of a filamentous fungus.

\section{Conflict of interest}

482 The authors disclose no potential conflicts of interest. Both authors approved the final article.

\section{Author contributions}


485 P.H-O. conducted the experiments, analyzed the data and wrote the manuscript. E.A.E. designed 486 the study, supervised the research and wrote the manuscript.

487

488 Acknowledgements

489 This work was supported by the Spanish Ministerio de Economia y Competitividad [Grants 490 BFU2012-33142 and BFU2015-66806-R to E.A.E.] and by the Spanish Research Council 491 Consejo Superior de Investigaciones Cientificas [JAE-Pre fellowship to P.H-O]. We wish to 492 thank Dr Oier Etxebeste for critical reading of the manuscript and for providing us with the 493 CaM-GFP strain. Also to Dr. Sotiris Amillis for the VIE172 strain. We are grateful to Elena 494 Reoyo for her assistance and technical advice. 
497 Fig. 1. Cellular localization of components of the calcium signaling machinery. Fluorescence 498 images of cellular localization of GFP-tagged proteins CrzA, CaM, CnaA, CkiA and GskA. 499 Insets and magnified images show in detail the apex (marked with asterisk), septa (s, white 500 arrowhead) (also Normarski images) and nuclei (n, arrow) of the cells. Also indicated is the 501 cytoplasmic accretion of CaM-GFP, black arrowhead. Strains used in this experiment are, top to bottom: MAD3021, MAD4310, MAD4366, VIE172 and MAD4643. Scale bar, $5 \mu \mathrm{m}$.

503

Fig. 2. Analysis of cellular localization of $\mathrm{CaM}$, CnaA, CkiA and GskA in response to stress. (A) Conidiospores from the $c r z A-g f p$ strain (MAD3021) and the $c a M-g f p$ strain (MAD4310) CrzA in nuclei, $\mathrm{CrzA}_{\text {nuc }}$, while did not induce major changes in the cellular location of CnaA. Inset images display fluorescence of CnaA-GFP along the hyphae (left) and at the apex (right). (C) Strain VIE172 did not exhibit any change in the cellular distribution of CkiA-GFP after

514 stress induction. (D) Cell from strain MAD4643 shows that GskA-GFP (green) did not co515 localize with the nuclear marker histone H1, HhoA (red), and maintained this distribution in the 516 presence of stress. For all experiments in this figure: $\mathrm{RC}$, resting conditions; $\mathrm{Ca}^{2+}, 50 \mathrm{mM}$ $517 \mathrm{CaCl}_{2} ; \mathrm{pH} 8,100 \mathrm{mM} \mathrm{Na} 2 \mathrm{HPO}_{4}$. Scale bar, $5 \mu \mathrm{m}$.

Fig. 3. Effects of different concentrations of calcium and manganese in the signaling of CrzA. (A) Growth tests of CrzA-GFP (MAD3021) and null crzA (MAD3709) strains. Strains were 521 inoculated on standard AMM (-) and AMM supplemented with increasing concentrations (mM) of $\mathrm{Ca}^{2+}\left(\mathrm{CaCl}_{2}\right)$. Images of colonies were taken after 2 days of incubation at $37^{\circ} \mathrm{C}$. 
523 Electrophoretic mobility of CrzA-GFP in protein extracts from strain MAD3021 after addition

524 of calcium $\left(\mathrm{Ca}^{2+}\right)$. The concentration of $\mathrm{CaCl}_{2}$ used in each assay is indicated in $\mathrm{mM}$ on top of

525 the image and mycelium samples of each condition were taken at the indicated times (min). (C,

526 D and E) Conidiospores from strain MAD4641 were grown in $\mathrm{WMM}$ at $25^{\circ} \mathrm{C}$ for $18 \mathrm{~h}$ and

527 observed by fluorescence microscopy under different stress conditions.(C) CrzA was nuclear for

528 at least $30 \mathrm{~min}$ in cells treated with $50 \mathrm{mM} \mathrm{Ca}^{2+}$ or $10 \mathrm{mM} \mathrm{Mn}^{2+}$. (D) Initially (RC) CrzA is

529 excluded from nuclei. After addition of $1 \mathrm{mM} \mathrm{CaCl}_{2}, \mathrm{CrzA}$ accumulated in nuclei from minute

5301 and remained in this location for at least 30 minutes. (E) Addition of $1 \mathrm{mM} \mathrm{MnCl}_{2}$ caused a

531 temporal accumulation of CrzA in nuclei. Scale bars, $5 \mu \mathrm{m}$

532

533 Fig. 4. Effects of medium alkalinisation in the signaling of CrzA. (A, B and C) Conidiospores 534 from strain MAD4641 were grown in WMM at $25^{\circ} \mathrm{C}$ for $18 \mathrm{~h}$ and observed by fluorescence 535 microscopy after addition of different buffering agents. (A) $100 \mathrm{mM} \mathrm{Na} \mathrm{HPO}_{4}$ generates a 536 transient nuclear accumulation of CrzA. (B) $10 \mathrm{mM}$ Tris- $\mathrm{HCl} \mathrm{pH} 8$ caused a similar effect to 537 that observed with $\mathrm{Na}_{2} \mathrm{HPO}_{4}$ buffer. (C) A tenfold reduction in $\mathrm{Na}_{2} \mathrm{HPO}_{4}$ concentration, still 538 maintaining the buffering effect at $\mathrm{pH} 8$ in WMM, caused a short and partial pulse of nuclear 539 accumulation of CrzA. RC, resting conditions.

540

541 Fig. 5. Differential tip-to-base nuclear accumulation of $\mathrm{CrzA}^{\Delta \mathrm{CDD} 2}$. Conidiospores from the strain 542 MAD4234 expressing a GFP-tagged version of CrzA lacking the CDD2 were cultured in WMM 543 for $18 \mathrm{~h}$ at $25^{\circ} \mathrm{C}$. In resting cells (RC), basal conditions, CrzA remained in the cytoplasm. In 544 cells shifted to fresh WMM supplemented with $\mathrm{Ca}^{2+}\left(50 \mathrm{mM} \mathrm{CaCl}_{2}\right)$, the recruitment of CrzA to 545 the nucleus most proximal to the apex (white arrowhead) was visible after 5 minutes of stress 546 induction. Subsequently, nuclear recruitment of CrzA spread towards the base of cell 547 compartment. The white arrow points to a nucleus distant to the cell tip. Insets show the 548 dynamics of CrzA transport in the apical and basal nuclei. The white star indicates the tip of a 549 cell. Scale bar, $5 \mu \mathrm{m}$ 
551 Fig. 6. Low calcium concentration promotes a spatiotemporal nuclear recruitment of CrzA.

552 Cells of the $c r z A$-gfp strain MAD3021 were grown in WMM for $18 \mathrm{~h}$ at $25^{\circ} \mathrm{C}$. In the absence of 553 stimulus $(0 \mathrm{~min}), \mathrm{CrzA}$ is distributed along the cytoplasm. 1 minute after addition of $10 \mu \mathrm{M}$

$554 \mathrm{CaCl}_{2}, \mathrm{CrzA}-\mathrm{GFP}$ fluorescence accumulated in the apical nucleus. Nuclear recruitment of CrzA was propagated to the rest of nuclei from the tip towards the base of the cell. The white star indicates the position of the tip. Arrowheads point to nuclei, numbered 1, 3 and 5 for their first, third and fifth position relative to the tip. On the right, magnification of nuclei 1,3 and 5 organized from minute 0 to 30 . Scale bar, $5 \mu \mathrm{m}$

Fig. 7. Effects of disruption of microtubule and actin cytoskeletons in the signaling of CrzA.

(A) Conidiospores of the $c r z A-g f p$ strain MAD3021 were grown in WMM for $18 \mathrm{~h}$ at $25^{\circ} \mathrm{C}$.

Cells were incubated in the presence of $4.8 \mu \mathrm{g} / \mathrm{ml}$ benomyl (Ben) during 20 minutes in order to ensure microtubule polymerization disruption, as it is observed as swelling in the tips and subsequent stop of apical growth. After this initial treatment, cells were imaged after addition of $50 \mathrm{mM} \mathrm{CaCl}_{2}\left(\mathrm{Ca}^{2+}\right)$ or $100 \mathrm{mM} \mathrm{Na}_{2} \mathrm{HPO}_{4}(\mathrm{pH} 8)$. Presence of the drug did not alter the nuclear entry of CrzA-GFP after stress induction. The white star indicates the tip. (B) Strains MAD4310 (CaM-GFP) and MAD4366 (CnaA-GFP) were grown and treated with benomyl as in (A). Black arrows indicate the localization of fluorescent $\mathrm{CaM}$ and $\mathrm{CnaA}$ fusions in each strains in cells showing tip swelling and recovery of polar growth. Grey arrows point to the swelling area of each cell generated by benomyl treatment. (C) Conidiospores from strains MAD4310 (CaMGFP), MAD4366 (CnaA-GFP), MAD3021 (CrzA-GFP) and MAD3222 (CrzA-GFP cnaAA) were inoculated along with conidiospores from the strain MAD1399 (AbpA-mRFP) in the same chamber containing WMM and incubated for $18 \mathrm{~h}$ at $25^{\circ} \mathrm{C}$. Images were taken before and after 574 treatment with $100 \mu \mathrm{M}$ Latrunculin B (LatB) for 20 minutes. The white star indicates the tip at 575 the cell expressing AbpA-mRFP. (C) Conidiospores from the $c r z A-g f p$ strain (MAD3021) and the $a b p A-m r f p$ strain (MAD1399) were co-inoculated in WMM and incubated for $18 \mathrm{~h}$ at $25^{\circ} \mathrm{C}$. 
577 After the initial period of growth in resting conditions (RC), cells were treated with $4.8 \mu \mathrm{g} / \mathrm{ml}$ 578 benomyl for 20 minutes (+Ben). Subsequently, $100 \mu \mathrm{M}$ Latrunculin B was added to the same 579 culture and incubated for 20 minutes. The white star indicates the tip at the cell expressing 580 AbpA-mRFP. Scale bars, $5 \mu \mathrm{m}$.

581

582 Fig. 8. Tentative models of CrzA regulation and nuclear transport in $A$. nidulans. (A) Model 583 combining posttranslational modifications in CrzA and nuclear transport. Differential localization of casein kinase I, CkiA, and glycogen synthase kinase $3 \beta$, GskA, predict for CkiA a role in nucleus for phosphorylation of CrzA and promoting nuclear export, and for GskA in 586 regulating the different levels of CrzA phosphorylation at the cytoplasm

587 (Activation/inactivation step, labeled with number 1). Low level or non-phosphorylated CrzA 588 forms, generated by the $\mathrm{CN}$ phosphatase activity, are transported in the nucleus. In contrast, 589 phosphorylated forms, hypothetically generated by CkiA activity, are preferentially exported to 590 the cytoplasm. The identity of exportins (exp) and importins (imp) involved remain unknown in 591 A. nidulans (regulation of nuclear transport step, labeled with number 2). Nuclear CrzA 592 regulates transcription of genes required for the response to calcium, manganese and alkaline

$593 \mathrm{pH}$ stresses, among other stimuli (transcriptional regulation step, labeled with number 3) (B) A 594 schematic representation of part of an apical compartment in a vegetative cell. Vesicles 595 accumulate at the tip due to a continuous feed of exocytic vesicles, some of which are originated 596 at the endocytic ring where AbpA protein locates. The Splitzenkorper (SPK) is formed at the 597 apex by a continuous supplementation of vesicles, being structurally supported by the actin 598 cytoskeleton and other associated protein complexes. Calcineurin $(\mathrm{CN})$ and calmodulin $(\mathrm{CaM})$ 599 accumulate at the SPK. CRZ factors become activated by $\mathrm{CN}$, possibly after a rise of 600 cytoplasmic calcium levels. Calcium may enter the cell by an excess of extracellular 601 concentration through channels or transporters located at the plasma membrane (PM). 602 Alkalinity and $\mathrm{Mn}^{2+}$ may activate those $\mathrm{PM} \mathrm{Ca}^{2+}$-transporters or cause the release of $\mathrm{Ca}^{2+}$ from 
603

604

605

606

607

608

609

610

611

612

613

614

615

616

617

618

619

620

621

622

623

624

625

626

627

628

629

630

631

632

633

634

635

636

637

638

internal stores. Numbers indicate the three predicted steps for activation and functional role of CRZ proteins in most fungi as indicated in panel A.

References

[1] M J Berridge, P. Lipp, M.D. Bootman, The versatility and universality of calcium signalling, Nat. Rev. Mol. Cell Biol. 1 (2000) 11-21.

[2] D Chin and A.R. Means, Calmodulin: a prototypical calcium sensor, Trends Cell Biol. 10 (2000) 322-328.

[3] D E Clapham, Calcium signaling, Cell 131 (14-12-2007) 1047-1058.

[4] P Kursula, The many structural faces of calmodulin: a multitasking molecular jackknife, Amino. Acids (9-7-2014)

[5] D Guerini, Calcineurin: not just a simple protein phosphatase, Biochem. Biophys. Res. Commun. 235 (18-6-1997) 271-275.

[6] F Rusnak and P. Mertz, Calcineurin: form and function, Physiol Rev. 80 (2000) 14831521.

[7] A Rodríguez, J. Roy, S. Martínez-Martínez, M.D. López-Maderuelo, P. Nino-Moreno, L. Orti, D. Pantoja-Uceda, A. Pineda-Lucena, M.S. Cyert, J.M. Redondo, A conserved docking surface on calcineurin mediates interaction with substrates and immunosuppressants, Mol. Cell 33 (13-3-2009) 616-626.

[8] J Aramburu, F. García-Cozar, A. Raghavan, H. Okamura, A. Rao, P.G. Hogan, Selective inhibition of NFAT activation by a peptide spanning the calcineurin targeting site of NFAT, Mol. Cell 1 (1998) 627-637.

[9] M S Cyert, Calcineurin signaling in Saccharomyces cerevisiae: how yeast go crazy in response to stress, Biochem. Biophys. Res. Commun. 311 (28-11-2003) 1143-1150.

[10] S Feske, A. Rao, P.G. Hogan, The $\mathrm{Ca}^{2+}$-calcineurin-NFAT signalling pathway, (2007)

[11] S Thewes, Calcineurin-Crz1 signaling in lower eukaryotes, Eukaryot. Cell 13 (2014) 694-705.

[12] G R Crabtree and E.N. Olson, NFAT signaling: choreographing the social lives of cells, Cell 109 Suppl (2002) S67-S79.

[13] M Mancini and A. Toker, NFAT proteins: emerging roles in cancer progression, Nat. Rev. Cancer 9 (2009) 810-820.

[14] D S Fox and J. Heitman, Good fungi gone bad: the corruption of calcineurin, Bioessays 24 (2002) 894-903. 
[15] P Hernández-Ortiz and E.A. Espeso, Phospho-regulation and nucleocytoplasmic trafficking of CrzA in response to calcium and alkaline-pH stress in Aspergillus nidulans, Mol. Microbiol. 89 (2013) 532-551.

[16] K A Kafadar, H. Zhu, M. Snyder, M.S. Cyert, Negative regulation of calcineurin signaling by Hrr25p, a yeast homolog of casein kinase I, Genes Dev. 17 (1-11-2003) 2698-2708.

[17] K A Kafadar and M.S. Cyert, Integration of stress responses: modulation of calcineurin signaling in Saccharomyces cerevisiae by protein kinase A, Eukaryot. Cell 3 (2004) 1147-1153.

[18] M Riquelme, Tip growth in filamentous fungi: a road trip to the apex, Annu. Rev. Microbiol. 67 (2013) 587-609.

[19] S Chen, Y. Song, J. Cao, G. Wang, H. Wei, X. Xu, L. Lu, Localization and function of calmodulin in live-cells of Aspergillus nidulans, Fungal. Genet. Biol. 47 (2010) 268278.

[20] P R Juvvadi, J.R. Fortwendel, L.E. Rogg, K.A. Burns, S.H. Randell, W.J. Steinbach, Localization and activity of the calcineurin catalytic and regulatory subunit complex at the septum is essential for hyphal elongation and proper septation in Aspergillus fumigatus, Mol. Microbiol. 82 (2011) 1235-1259.

[21] G Wang, L. Lu, C.Y. Zhang, A. Singapuri, S. Yuan, Calmodulin concentrates at the apex of growing hyphae and localizes to the Spitzenkorper in Aspergillus nidulans, Protoplasma 228 (2006) 159-166.

[22] A Apostolaki, L. Harispe, A.M. Calcagno-Pizarelli, I. Vangelatos, V. Sophianopoulou, H.N. Arst, Jr., M.A. Peñalva, S. Amillis, C. Scazzocchio, Aspergillus nidulans CkiA is an essential casein kinase I required for delivery of amino acid transporters to the plasma membrane, Mol. Microbiol. 84 (2012) 530-549.

[23] H S Kim, K.J. Czymmek, A. Patel, S. Modla, A. Nohe, R. Duncan, S. Gilroy, S. Kang, Expression of the Cameleon calcium biosensor in fungi reveals distinct $\mathrm{Ca}(2+)$ signatures associated with polarized growth, development, and pathogenesis, Fungal. Genet. Biol. 49 (2012) 589-601.

[24] T Nayak, E. Szewczyk, C.E. Oakley, A. Osmani, L. Ukil, S.L. Murray, M.J. Hynes, S.A. Osmani, B.R. Oakley, A versatile and efficient gene-targeting system for Aspergillus nidulans, Genetics 172 (2006) 1557-1566.

[25] M A Peñalva, Tracing the endocytic pathway of Aspergillus nidulans with FM4-64, Fungal. Genet. Biol. 42 (2005) 963-975.

[26] P R Juvvadi, J.R. Fortwendel, N. Pinchai, B.Z. Perfect, J. Heitman, W.J. Steinbach, Calcineurin localizes to the hyphal septum in Aspergillus fumigatus: implications for septum formation and conidiophore development, Eukaryot. Cell 7 (2008) 1606-1610.

[27] M J Egan, M.A. McClintock, S.L. Reck-Peterson, Microtubule-based transport in filamentous fungi, Curr. Opin. Microbiol. 15 (2012) 637-645.

[28] N Taheri-Talesh, T. Horio, L. Araújo-Bazán, X. Dou, E.A. Espeso, M.A. Peñalva, S.A. Osmani, B.R. Oakley, The tip growth apparatus of Aspergillus nidulans, Mol. Biol. Cell 19 (2008) 1439-1449. 
[29] L Araújo-Bazán, M.A. Peñalva, E.A. Espeso, Preferential localization of the endocytic internalization machinery to hyphal tips underlies polarization of the actin cytoskeleton in Aspergillus nidulans, Mol. Microbiol. 67 (2008) 891-905.

[30] A Markina-Iñarrairaegui, O. Etxebeste, E. Herrero-Garcia, L. Araújo-Bazán, J. Fernández-Martínez, J.A. Flores, S.A. Osmani, E.A. Espeso, Nuclear transporters in a multinucleated organism: functional and localization analyses in Aspergillus nidulans, Mol. Biol. Cell 22 (2011) 3874-3886.

[31] H C Li and W.W. Chan, Activation of brain calcineurin towards proteins containing $\operatorname{Thr}(\mathrm{P})$ and $\operatorname{Ser}(\mathrm{P})$ by $\mathrm{Ca} 2+$, calmodulin, $\mathrm{Mg} 2+$ and transition metal ions, Eur. J. Biochem. 144 (2-11-1984) 447-452.

[32] M S Cyert and C.C. Philpott, Regulation of cation balance in Saccharomyces cerevisiae, Genetics 193 (2013) 677-713.

[33] H Wang, Y. Liang, B. Zhang, W. Zheng, L. Xing, M. Li, Alkaline stress triggers an immediate calcium fluctuation in Candida albicans mediated by Rim101p and Crz1p transcription factors, FEMS Yeast Res. 11 (2011) 430-439.

[34] D Hagiwara, A. Kondo, T. Fujioka, K. Abe, Functional analysis of C2H2 zinc finger transcription factor CrzA involved in calcium signaling in Aspergillus nidulans, Curr. Genet. 54 (2008) 325-338.

[35] A Spielvogel, H. Findon, H.N. Arst, Jr., L. Araújo-Bazán, P. Hernández-Ortiz, U. Stahl, V. Meyer, E.A. Espeso, Two zinc finger transcription factors, CrzA and SltA, are involved in cation homoeostasis and detoxification in Aspergillus nidulans, Biochem. $\mathrm{J}$ 414 (15-9-2008) 419-429.

[36] S Kim, J. Hu, Y. Oh, J. Park, J. Choi, Y.H. Lee, R.A. Dean, T.K. Mitchell, Combining ChIP-chip and expression profiling to model the MoCRZ1 mediated circuit for $\mathrm{Ca} /$ calcineurin signaling in the rice blast fungus, PLoS. Pathog. 6 (2010) e1000909-

[37] L J Lombardia, M. Becerra, E. Rodriguez-Belmonte, N.C. Hauser, M.E. Cerdan, Genome-wide analysis of yeast transcription upon calcium shortage, Cell Calcium 32 (2002) 83-91.

[38] F M Soriani, I. Malavazi, M. Savoldi, E. Espeso, T.M. Dinamarco, L.A. Bernardes, M.E. Ferreira, M.H. Goldman, G.H. Goldman, Identification of possible targets of the Aspergillus fumigatus CRZ1 homologue, CrzA, BMC. Microbiol. 10 (2010) 12-

[39] C R Beals, C.M. Sheridan, C.W. Turck, P. Gardner, G.R. Crabtree, Nuclear export of NF-ATc enhanced by glycogen synthase kinase-3, Science 275 (28-3-1997) 1930-1934.

[40] Z Hilioti, D.A. Gallagher, S.T. Low-Nam, P. Ramaswamy, P. Gajer, T.J. Kingsbury, C.J. Birchwood, A. Levchenko, K.W. Cunningham, GSK-3 kinases enhance calcineurin signaling by phosphorylation of RCNs, Genes Dev. 18 (1-1-2004) 35-47.

[41] F V Rivas, J.P. O'Keefe, M.L. Alegre, T.F. Gajewski, Actin cytoskeleton regulates calcium dynamics and NFAT nuclear duration, Mol. Cell Biol. 24 (2004) 1628-1639.

[42] Y Wang, M.P. Mattson, K. Furukawa, Endoplasmic reticulum calcium release is modulated by actin polymerization, J. Neurochem. 82 (2002) 945-952.

[43] E Herrero-Garcia, E. Perez-de-Nanclares-Arregi, M.S. Cortese, A. MarkinaIñarrairaegui, E. Oiartzabal-Arano, O. Etxebeste, U. Ugalde, E.A. Espeso, Tip-to- 
nucleus migration dynamics of the asexual development regulator FlbB in vegetative cells, Mol. Microbiol. (10-8-2015)

725

726

727

728

729

730

731

732

733

[44] O Bayram, O.S. Bayram, Y.L. Ahmed, J. Maruyama, O. Valerius, S.O. Rizzoli, R. Ficner, S. Irniger, G.H. Braus, The Aspergillus nidulans MAPK module AnSte11Ste50-Ste7-Fus3 controls development and secondary metabolism, PLoS. Genet. 8 (2012) e1002816-

[45] E Bielska, Y. Higuchi, M. Schuster, N. Steinberg, S. Kilaru, N.J. Talbot, G. Steinberg, Long-distance endosome trafficking drives fungal effector production during plant infection, Nat. Commun. 5 (2014) 5097- 
Figure 1
Click here to download high resolution image
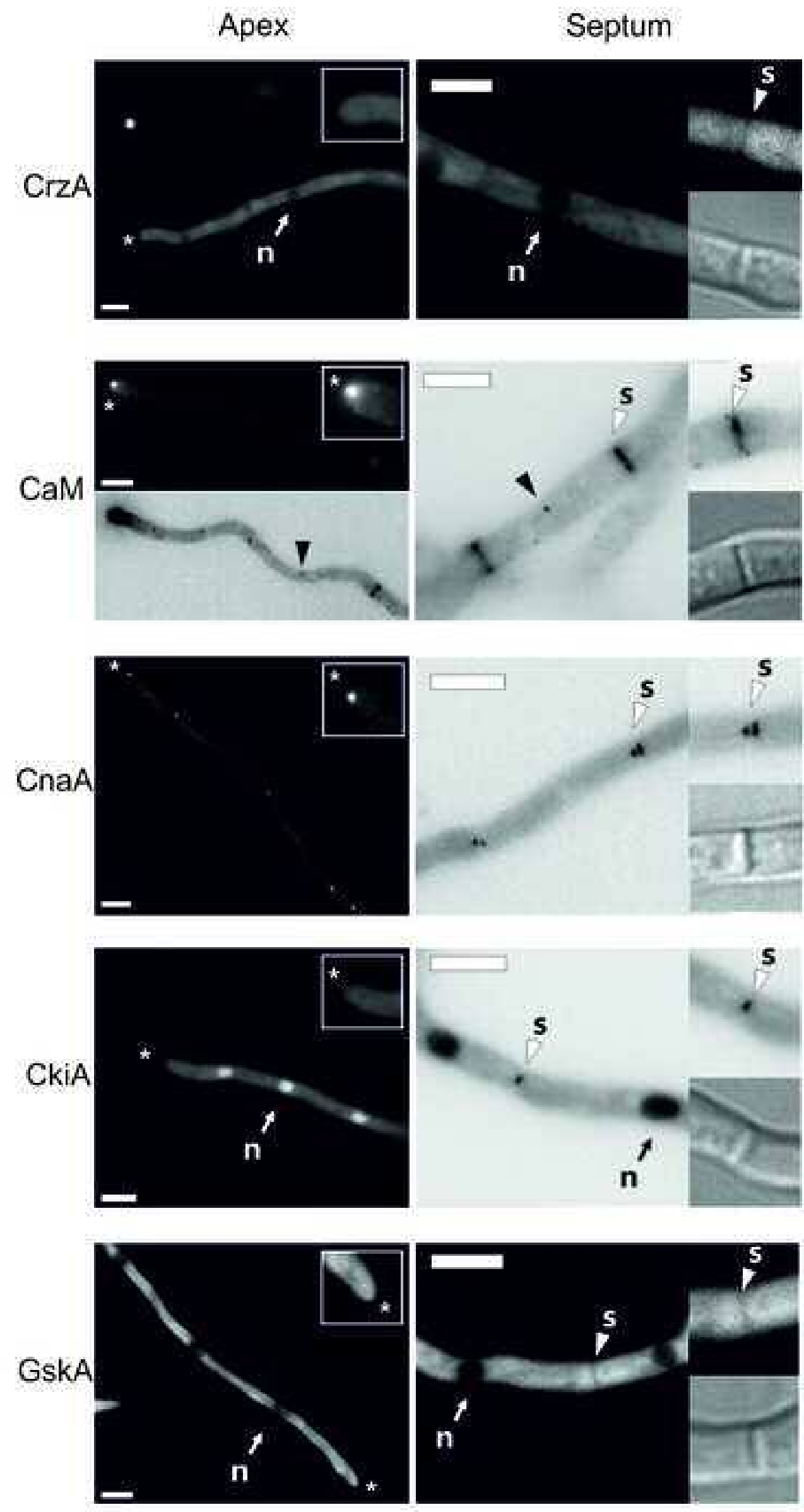


\section{Figure 2}

Click here to download high resolution image

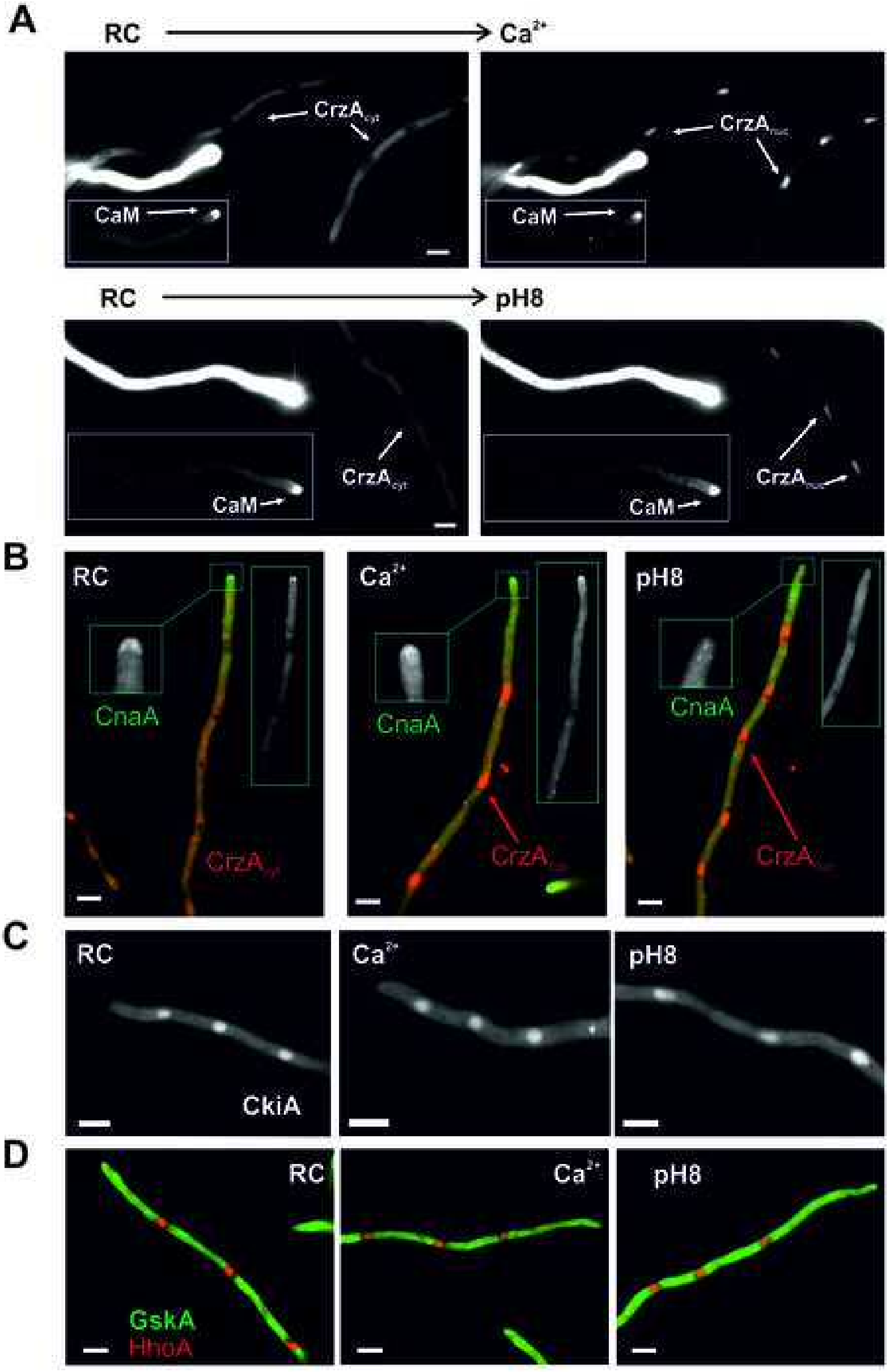


Click here to download high resolution image

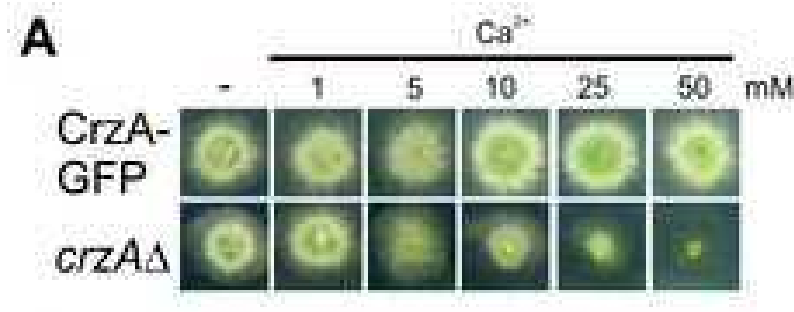

B CrZA-GFP

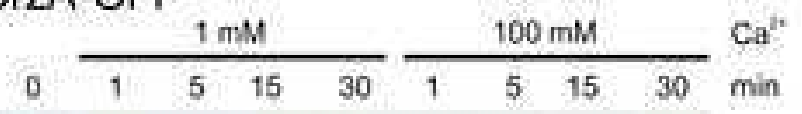

P.CrzA-

C
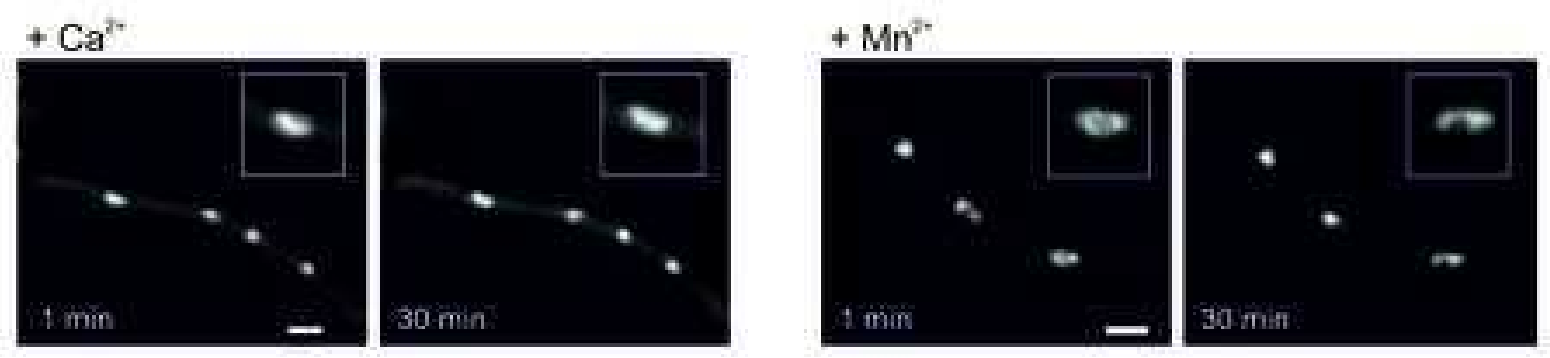

D
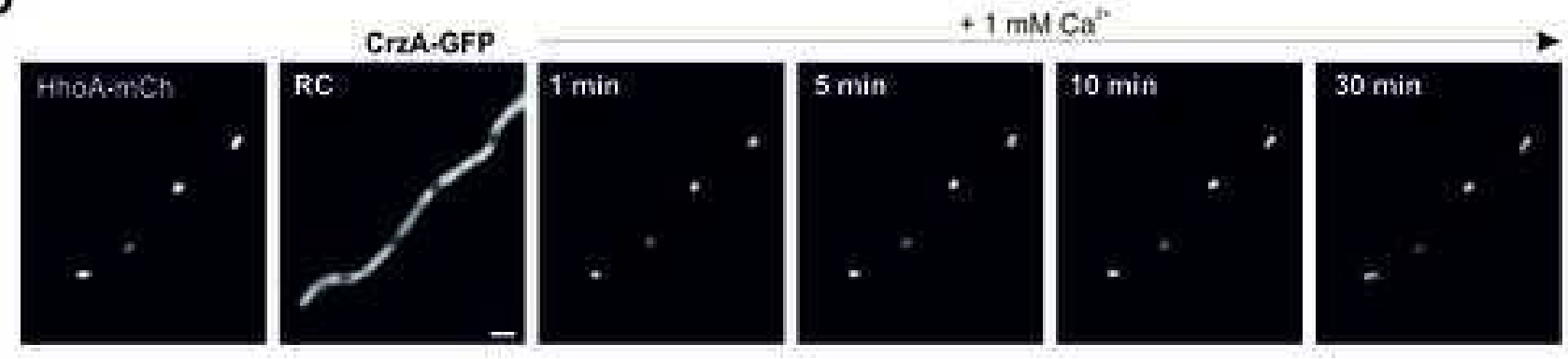

E
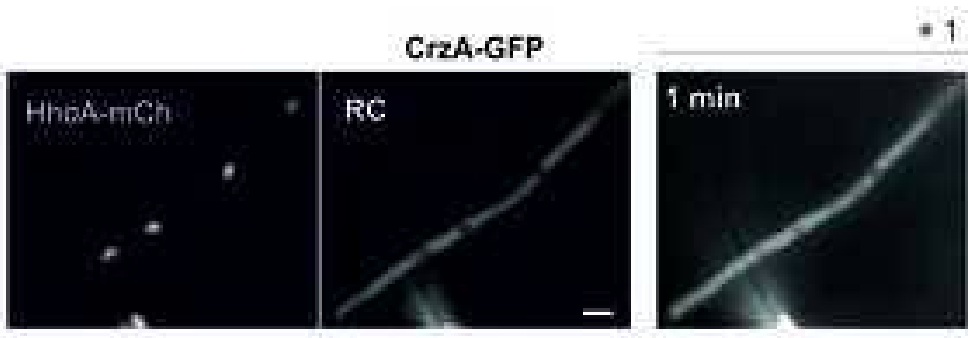

$1 \mathrm{mM} \mathrm{Mn}{ }^{2 *}$
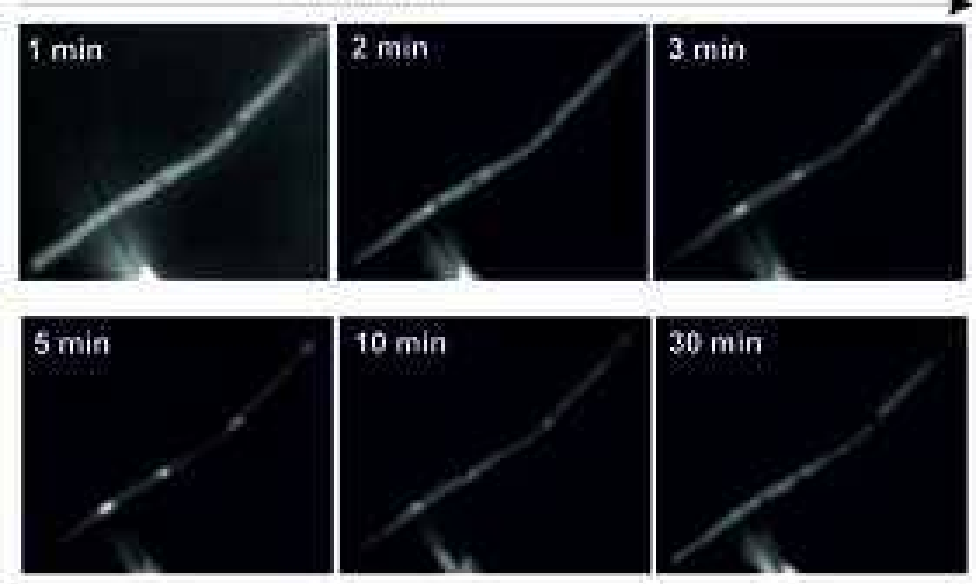

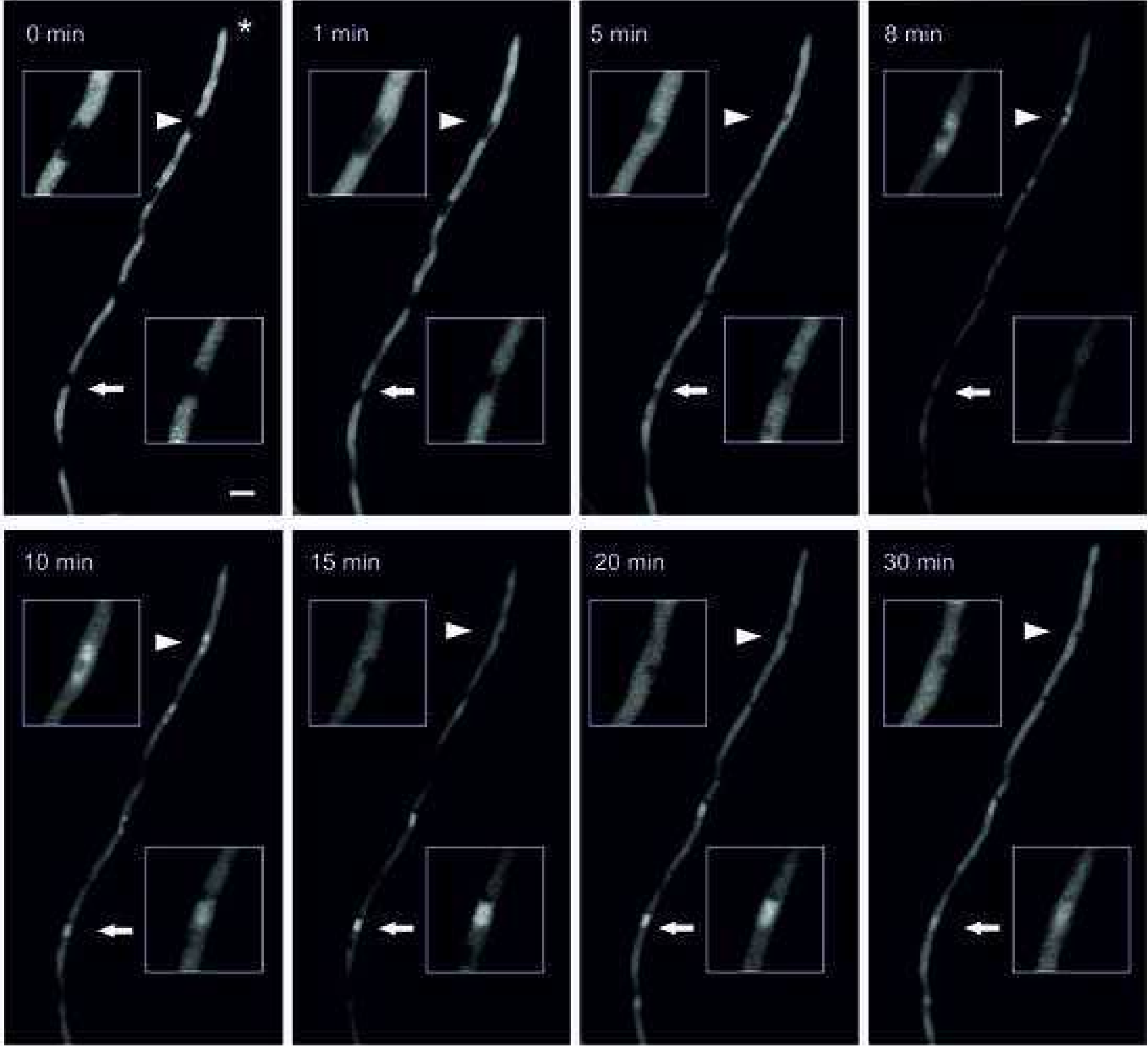
Flick here to download high resolution image
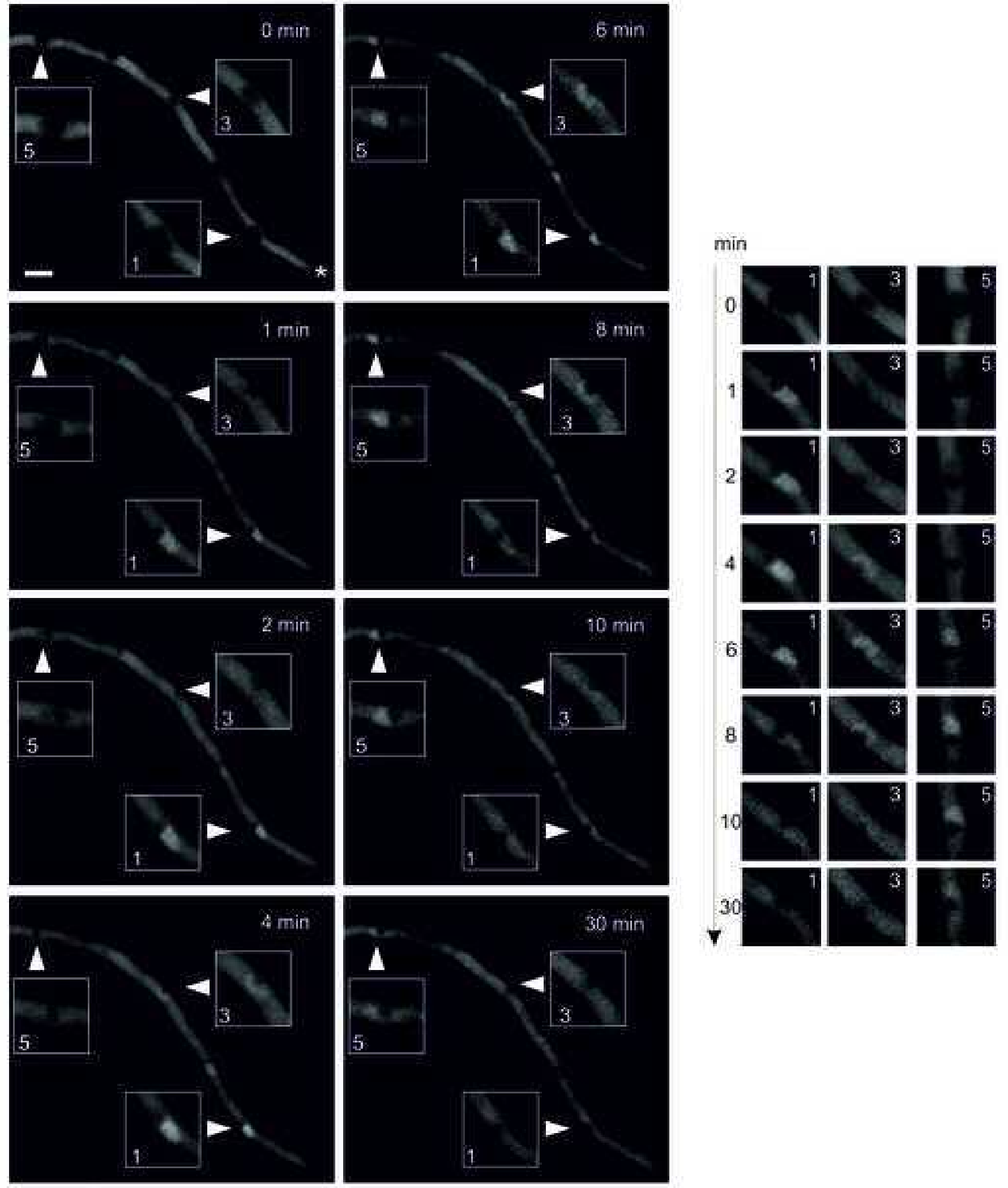
Figure 7

Click here to download high resolution image
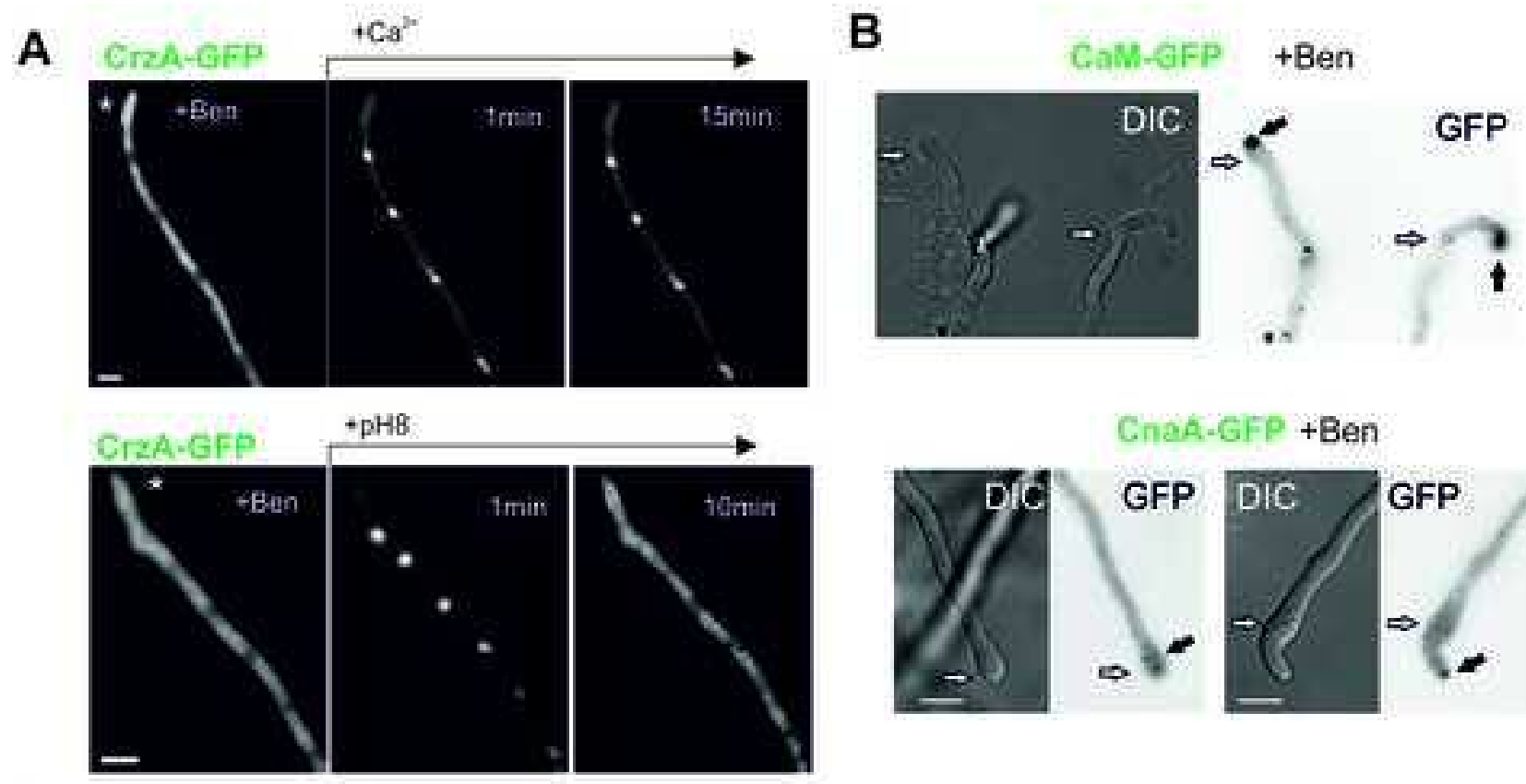

C

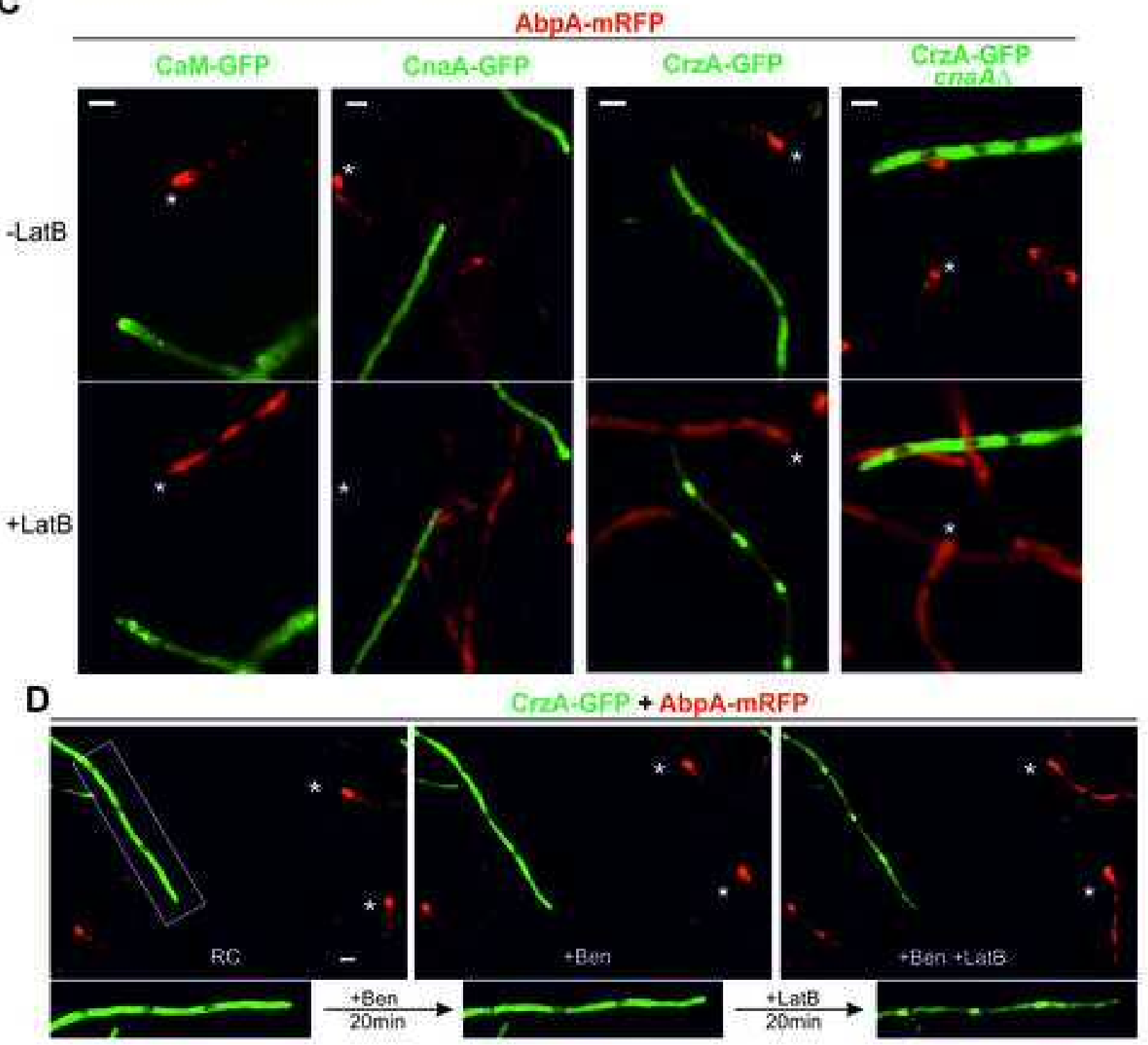


A

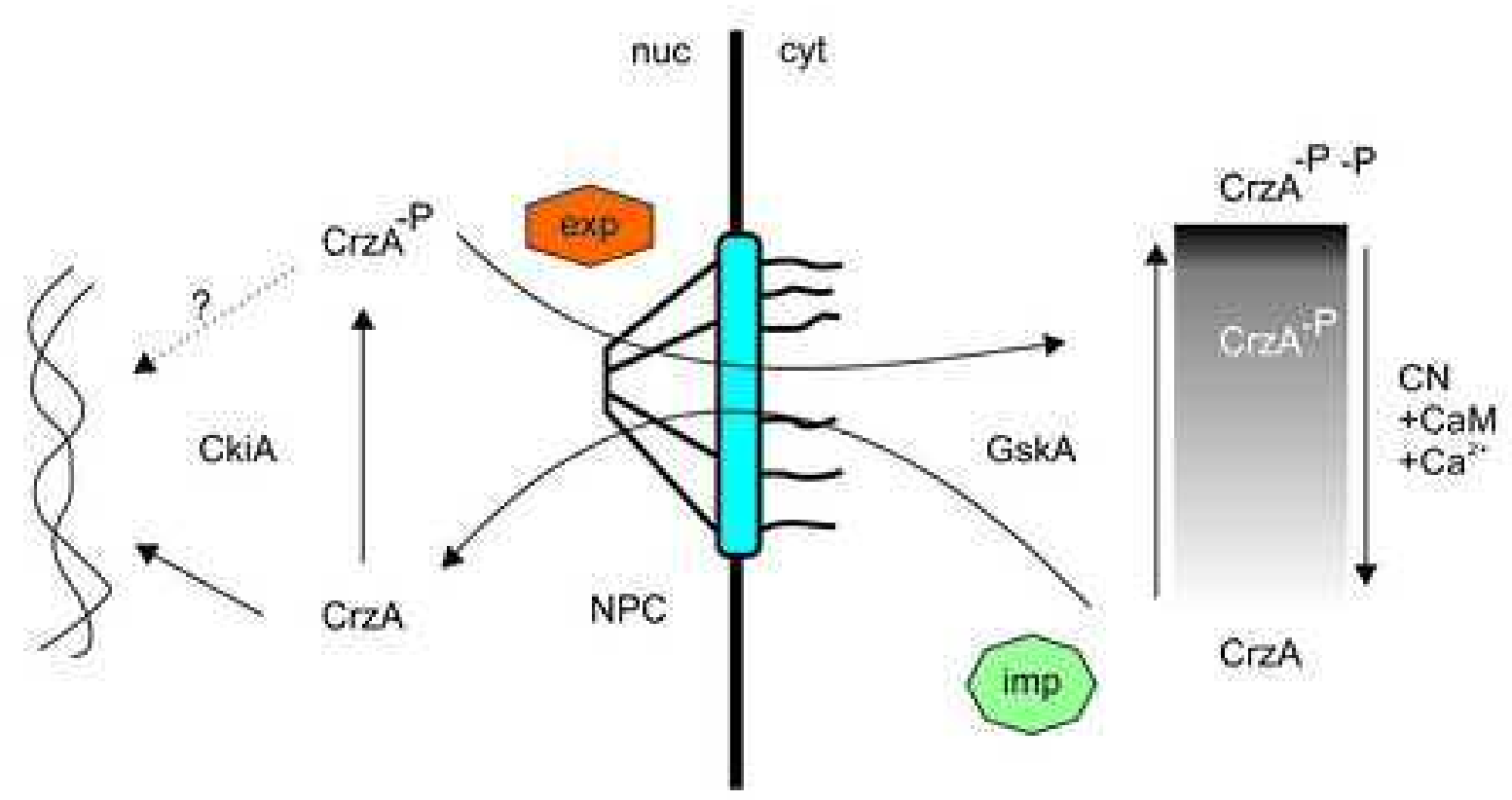

Transcriptional
regulation

Regulation of nuclear transport

(2)

Activation / Inactivation

(1)

B

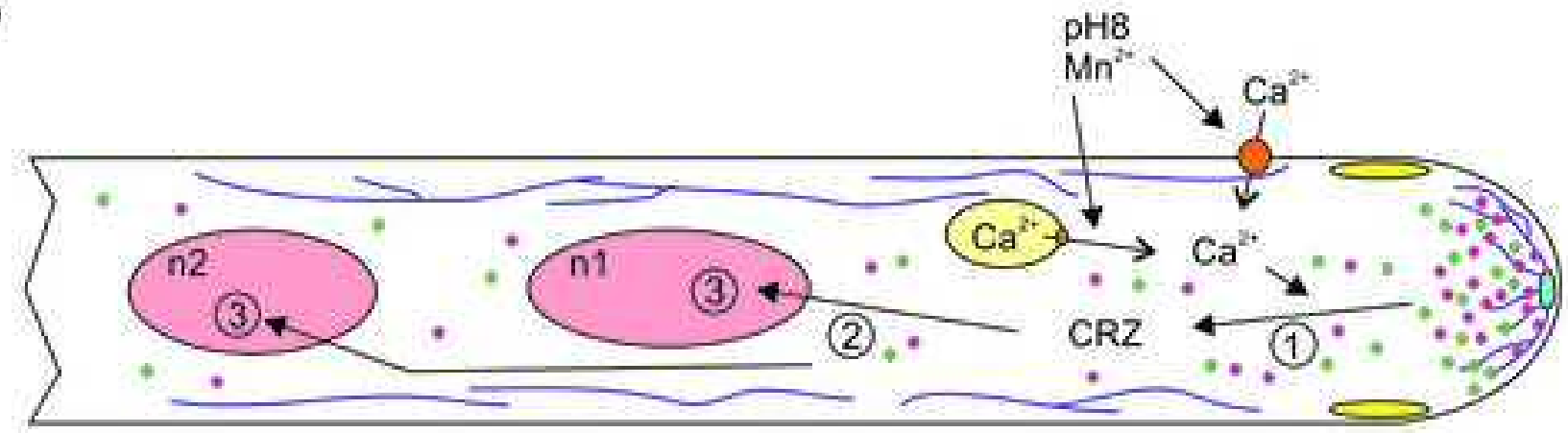

SPK

0

F-actin

Endocytosis

Ring

Nucleus

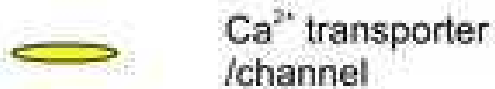

Ichannel

Intracellular $\mathrm{Ca}^{24}$ store
Calmodulin (CaM)

Calcineurin ( $\mathrm{CN}$ ) 
TABLE 1. List of strains used in this work

\begin{tabular}{|c|c|c|}
\hline Strain & Relevant genotype & Source \\
\hline MAD1399 & abpA-mrfp::pyrG $G^{A f}$ & (3) \\
\hline MAD2666 & wild type & $(36)$ \\
\hline MAD2446 & hhoA::mCh::pyro $A^{A f}$ & $(15)$ \\
\hline MAD3021 & $\operatorname{crzA}: \because g f p:: p y r G^{A f}$ & (23) \\
\hline MAD3222 & cnaAA::pyro $A^{A f} ;$ crzA::gfp::pyrG $G^{A f}$ & $(23)$ \\
\hline MAD3709 & crzA $\triangle:: r i b o B^{A f}$ & (23) \\
\hline MAD4234 & crzA(4M356-A372) $\because g f p: \because p y r G^{A f}$ & (23) \\
\hline MAD4310 & caM::gfp $:: p y r G^{A f}$ & $\begin{array}{l}\text { Etxebeste, O. Univ Basque } \\
\text { Country }\end{array}$ \\
\hline MAD4366 & cnaA::gfp::pyrG $G^{A f}$ & (23) \\
\hline MAD4522 & cnaA::gfp::pyrG ${ }^{A f} ;$ crzA::mCh::pyro $A^{A f}$ & This work \\
\hline MAD4641 & crzA::gfp::pyrG $G^{A f} ;$ hhoA::mCh::pyro $A^{A f}$ & (23) \\
\hline MAD4643 & gskA::gfp::pyrG $G^{A f} ;$ hhoA::mCh::pyro $A^{A f}$ & This work \\
\hline VIE172 & ckiA::gfp::pyrG $G^{A f}$ & (1) \\
\hline
\end{tabular}


TABLE 2. List of oligonucleotides used in this work

\begin{tabular}{|c|c|c|}
\hline Primer & Sequence 5' 3' & Purpose \\
\hline $\operatorname{CrzA}(3)$ & СTTTGCTACCTATTTCTCTGTTCAGTAGGG & \multirow{6}{*}{$\begin{array}{c}\text { Generation of } \\
\text { crzA::mCh-pyro } A^{A f} \\
\text { cassette }\end{array}$} \\
\hline $\operatorname{CrzA}(4)$ & CAACCCGCACCCCTACACTTCTCTACATCG & \\
\hline CrzA(5) & $\begin{array}{l}\text { GACTCCTCCAACAGAAGGCTATCCGAATG } \\
\text { G }\end{array}$ & \\
\hline $\operatorname{CrzA}(6)$ & TTTCCCCCAGTTCGGATTTGCGTAGCCAGC & \\
\hline $\operatorname{CrzA}\left(6^{\prime}\right)$ & $\begin{array}{l}\text { GCTGGCTACGCAAATCCGAACTGGGGGAA } \\
\text { AGGAGCTGGTGCAGGCGCTGGAGCC }\end{array}$ & \\
\hline $\operatorname{CrzA}\left(3^{\prime}\right)$ & $\begin{array}{l}\text { CCCTACTGAACAGAGAAATAGGTAGCAAA } \\
\text { GCTGTCTGAGAGGAGGCACTGATG }\end{array}$ & \\
\hline GskA(3) & GTCAACGTCATAGTGCTG & \multirow{6}{*}{$\begin{array}{c}\text { Generation of } \\
g s k A:: g f p:: p y r G^{A f} \\
\text { cassette }\end{array}$} \\
\hline GskA(4) & CGGTGATTGAGTGAAGTGTCC & \\
\hline GskA(5) & GACTCCTTCCCCGATCTTC & \\
\hline GskA(6) & GTCAAGGTGTGCCATCATTTC & \\
\hline GskA(6') & $\begin{array}{l}\text { GAAATGATGGCACACCTTGACGGAGCTGG } \\
\text { TGCAGGCGCTGGAGCCGGTGC }\end{array}$ & \\
\hline GskA(3') & $\begin{array}{l}\text { CAGCACTATGACGTTGACCTGTCTGAGAG } \\
\text { GAGGCACTGATG }\end{array}$ & \\
\hline
\end{tabular}




\section{Click here to download Supplementary Material: Fig S1.pdf}

,

(1) 
Supplementary Material Fig S2
Click here to download Supplementary Material: Fig S2rev.pdf

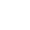
(1) (1)

- 
Click here to download Supplementary Material: Fig S3_oldS2.pdf

Supplementary Material Fig S3
Click here to download Suppler

\begin{abstract}
.
\end{abstract}

\author{
Fig S3_oldS2.pdf
}

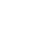

$\left(\frac{10}{20}\right.$

(a)

(1)

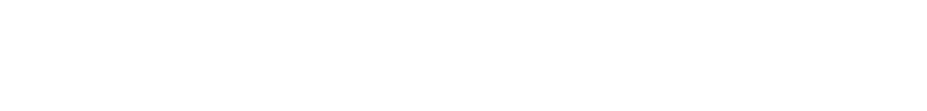

